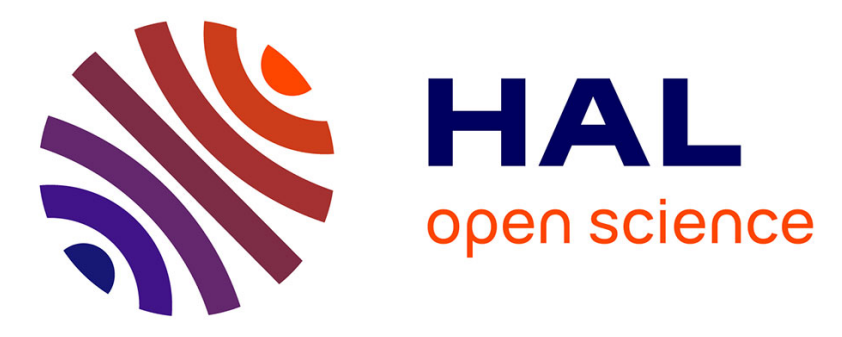

\title{
Coordinate free nonlinear incremental discrete mechanics
}

Jean Lerbet, Noël Challamel, François Nicot, Félix Darve

\section{To cite this version:}

Jean Lerbet, Noël Challamel, François Nicot, Félix Darve. Coordinate free nonlinear incremental discrete mechanics. Journal of Applied Mathematics and Mechanics / Zeitschrift für Angewandte Mathematik und Mechanik, 2018, 98 (10), pp.1813-1833. 10.1002/zamm.201700133 . hal-01857781

\section{HAL Id: hal-01857781 \\ https://hal.science/hal-01857781}

Submitted on 21 Nov 2018

HAL is a multi-disciplinary open access archive for the deposit and dissemination of scientific research documents, whether they are published or not. The documents may come from teaching and research institutions in France or abroad, or from public or private research centers.
L'archive ouverte pluridisciplinaire HAL, est destinée au dépôt et à la diffusion de documents scientifiques de niveau recherche, publiés ou non, émanant des établissements d'enseignement et de recherche français ou étrangers, des laboratoires publics ou privés. 


\title{
Coordinate free nonlinear incremental discrete mechanics
}

\author{
\begin{tabular}{l|l|l|l} 
J. Lerbet & | N. Challamel & | F. Nicot & | F. Darve
\end{tabular} \\ ${ }^{1}$ IBISC, Univ Evry, Université Paris-Saclay, 40 rue du Pelvoux CE 145591020 Evry Courcouronnes cedex, France \\ ${ }^{2}$ Université de Bretagne Sud UBS - IRDL-Centre de Recherche Rue de Saint Maudé - BP 9211656321 Lorient cedex, France \\ ${ }^{3}$ Grenoble Alpes University, IRSTEA, ETNA, Domaine universitaire, 38402 St Martin d'Heres cedex, France \\ ${ }^{4}$ Grenoble Alpes University, “Grenoble INP”, 3SR, BP 5338041 Grenoble cedex 9, France \\ Correspondence: J. Lerbet, IBISC, Univ Evry, Université Paris-Saclay, 40 rue du Pelvoux CE 145591020 Evry Courcouronnes cedex, \\ France. Email: jlerbet@gmail.com
}

In this paper we extend the Kinematic Structural Stability (KISS) issues obtained in the punctual linear case to a full non linear framework. Kinematic Structural Stability (KISS) refers to the stability of conservative or non-conservative elastic systems under kinematic constraints. We revisite and sometimes introduce some new mechanical and geometrically-related concepts, using the systematic language of vector bundles: transversality-stability, tangent stiffness tensor, loading paths and KISS. We bring an intrinsic geometrical meaning to these concepts and we extend the main results to a general nonlinear framework. The lack of connection on the configuration manifold in order to make derivatives of sections is bypassed thanks to the key rule of the nil section of the cotangent bundle. Two mechanical examples illustrate the interest of the new concepts, namely a discrete nonconservative elastic system under non-conservative loading (Ziegler's column) and, in more details, a four-sphere granular system with nonconservative interactions called diamond pattern.

KEYWORDS: coordinate free approach, fiber bundle, kinematic structural stability, transversality

\section{INTRODUCTION}

The initial aim of this work was to extend some of our recent results into the non linear differentiable framework but from an intrinsic geometrical point of view. We especially refer to the Kinematic Structural Stability (KISS) problem which has been the subject of numerous publications in the past years (see for example ${ }^{[5]}$ for the first partial result, ${ }^{[11]}$ for the general result for divergence linear stability, ${ }^{[13]}$ for a first geometrization, ${ }^{[14]}$ for the flutter KISS, ${ }^{[16]}$ for a full overview). All these works have been carried out within the framework of nonconservative linear discrete mechanics and dealt with the finite dimensional "punctual" issue which involved only tools of usual linear algebra. More recently ${ }^{[17]}$ performed the extension to continuous systems and involved then infinite dimension Hilbert spaces.

[12] and [15] proposed an extension to the nonlinear framework for the Geometric Degree of Nonconservativity (GDNC) issue and for its corresponding solution. But such an extension to the nonlinear framework for the KISS issue is still missing. The present paper address this problem. However, the extension to the non linear differentiable framework is not the only reason of this work.

We actually plan here to give an intrinsic and geometric formulation of the general corresponding mechanical issues and concepts. That means that the tools and the results must not depend on the parametrization of the mechanical problem or, in other more mathematical words, they do not depend on the choice of charts (or on an atlas) on the configuration manifold M. We then intend to carry out a geometric translation of the usual non intrinsic approach and to provide important new insights into concepts like the following usual ones: "tangent linear stiffness matrix", "loading path", "incremental loading path", "stability of an equilibrium" and so on.

Our language is the one of vector bundles especially applied here to the cotangent bundle of the configuration manifold. It is not the most common one for researchers and engineers in mechanics but it is the well adapted to our goal because it provides the appropriate geometrical framework. The mathematical objects involved in this work will then seem quite abstract but this 
abstraction is the cost to provide an intrinsic and geometrical translation of the above concepts involved in mechanics and to achieving our objective. To make the reading the most digestible possible, we propose to avoid proofs and / or calculations when they do not highlight the meaning of the results and we systematically illustrate our reasonings by pictures.

However, we must now warn the reader that the framework used hereafter is not the more general possible one. Indeed, in this paper, we imagine and investigate only quasi-static evolutions of the mechanical systems. Thus, no dynamics is taken into account. But, in order to make derivatives in a vector bundle, a connection (or a covariant derivative) is necessary. Whereas no natural connection emerges for quasi-static evolutions, the dynamic evolutions may use the Riemannian structure associated with the kinetic energy of the involved mechanical system. Rather, the lack of this natural connection on the vector bundle removes this natural way of making derivatives. Nevertheless, and this a central point of this work, we will be able to save the day by systematically taking advantage of the global nil section of the corresponding vector bundle.

We above mentioned that a part of the first claimed objective had been already carried out in [15] for the so-called GDNC issue which do not refer to dynamic nor quasi-static evolutions. A careful reader of this paper regarding GDNC will however note that no connection has been used. It is then worth noting that the derivative calculations have been done via the exterior derivative $\mathbf{d} \omega$ of the 1-form $\omega$ describing the set of forces acting on (and in) the considered mechanical system. This provides a skew-symmetric 2 -form $\mathbf{d} \omega$ without using any connection. This $(0,2)$ covariant skew symmetric tensor is actually the non-linear counterpart of the skew symmetric part of the usual stiffness matrix when the dynamic equations are linearized at an equilibrium configuration $m_{e} \in \mathbb{M}$. This surprising fact that no connection has been necessary comes from the remarkable fact that for ANY (symmetric) connection $\nabla$ like for example the connection attached to a Riemannian structure coming from kinetic energy, the skew-symmetric part of the covariant derivative $\nabla_{X} \omega$ is exactly the exterior derivative $\mathbf{d}$ :

$$
\nabla_{X} \omega(Y)-\nabla_{Y} \omega(X)=\mathbf{d} \omega(X, Y)
$$

for any vector fields $X, Y$ on $\mathbb{M}$. This explains why the general non linear framework of the skew symmetric part of the nonconservativeness has been more easily developed.

The paper is organized as follows. First, strong emphasis will be placed on describing a force system $\mathcal{F}$ on a mechanical system $\Sigma$. In the quasi-static (or incremental) analytic mechanic framework, it is a section $\omega_{\mathcal{F}}$ of the cotangent vector bundle $T^{*} \mathbb{M}$. In the following paragraph, we then focus on a quick presentation of the concepts attached to vector bundle geometry we will use. The third section is devoted to equilibriums and their stability. An intrinsic geometric definition of both an equilibrium and its stability is provided which does not use a prioiri any tangent stiffness tensor but uses the concept of transversality to a manifold. In order not to confuse this kind of stability with others already used in other contexts, we speak of Transversality stability or T- stability. In the fourth part, the corresponding infinitesimal aspects are tackled and calculations are carried out. The intrinsic counterpart of the usual tangent stiffness matrix is the vertical component of the differential of the section $\omega_{\mathcal{F}}$. This keeps meaningful without any connection thanks to the natural nil section of the cotangent bundle used somehow as an "horizontal" part. It is then proved that the geometric condition of Transversality stability can be translated into the invertibility of this tangent stiffness $(0,2)$ tensor. Hill's second order work criterion is then translated in this language and a first link between Transversality-stability and Hill's second order work criterion is given. A short fifth section is devoted to the translation in this geometric language of elasticity, hyperelasticity, hypoelasticity and incremental hypoelasticity. The following part focuses on quasi-static evolutions. We define loading paths $\mathcal{L}$ on $\mathbb{M}$ as couples $\mathcal{L}=\left(\mathbb{V}_{\mathcal{L}}, \omega_{\mathcal{L}}\right)$ where $\mathbb{V}_{\mathcal{L}}$ is a embedded submanifold of $\mathbb{M}$ and $\sigma \mapsto \omega_{\mathcal{L}}(\sigma)$ is a map from an interval $\left[\sigma_{1}, \sigma_{2}\right] \subset \mathbb{R}$ to $\Gamma\left(T^{*} \mathbb{V}_{\mathcal{L}}\right)$ set of sections of $T^{*} \bigvee_{\mathcal{L}}$. We then specify what mean regularity and singularity of $\mathcal{L}$ in relationship with the above Transversality Stability. The following part is devoted to the KISS issue. As for the linear "punctual" case tackled in our previous papers, Hill-stability exactly ensures the regularity for all loading paths. This last result is then the direct extension of the main result we obtained in the linear punctual framework. The eighth and last part applies the general developments to the example of the diamond pattern involved in granular mechanics. This system, naturally involving two charts on the configuration manifold, is particularly instructive to illustrate the general setting. Except this diamond pattern fully explored, throughout the text, when examples could help to understand the general reasonings and calculations, the paradigmatic Ziegler systems will be used. That allows us to also show how this work is the extension of the above mentioned previous works which illustrate the general results on such generic mechanical systems.To conclude this introduction, a short remark about the style of the paper is necessary. Readers who are comfortable with the langage of vector bundles obviously can bypass the concerning part of the text and of the numerous pictures mainly devoted to show the matching between this language and the mechanical issues. If the text can seem a little bit narrative, it is only to avoid a too dry approach and to convince a non mathematician reader of the value and of the meaning of this coordinate free geometric approach. This language is quite usual in analytic mechanics with, for example, the intensive use of symplectic geometry to investigate dynamics of Hamiltonian systems. It is interesting to note that the same language by means of other 
additional technics may be used, in this paper, to investigate the complete opposite framework of quasi-static non conservative systems.

\section{1 | FORCE SYSTEM AND LOAD SYSTEM}

The framework of this paper is analytic mechanics which means some hypotheses and some notations we now specify. The set $\mathbb{M}$ of configurations of the mechanical system $\Sigma$ is a $\mathcal{C}^{\infty} \mathrm{n}$-dimensional manifold. Any chart of $\mathbb{M}$ will be denoted by $\left(U_{\alpha}, \psi_{\alpha}\right)$ and the set $\left(\left(U_{\alpha}, \psi_{\alpha}\right)\right)_{\alpha}$ is an atlas of $\mathbb{M}$. For any $m \in \mathbb{M}$, its coordinates $\psi_{\alpha}(m)$ in this chart are denoted $q=\left(q^{1}, \ldots, q^{n}\right)$ but the dependency on $m$ is often not explicit. To present the problem, we will use such an atlas and in order not to complicate the text, we often do not distinct $m$ and $q$ with $m=\psi_{\alpha}(q)$ when $m \in U_{\alpha}$.

Our main hypothesis is that any physical action (internal or external as well, force or torque) symbolically written $F$ is only positional and then rate-independent. Indeed, because only quasi-static evolutions are investigated, the dependency on velocities of forces and interactions are purposeless. $\mathcal{F}=\{F\}$ is the set of forces also called the force system. Following the main idea of analytic mechanics, any "force" $F$ is thought as "something" (nobody knows what is a force!!) which, acting on any infinitesimal displacement, gives a scalar (the power of $F$ ) and linearly as function of this infinitesimal displacement. Any infinitesimal displacement at the configuration $m \in \mathbb{M}$ is the value $X(m)$ of a vector field $X$ on $\mathbb{M}$. Such a vector field $X$ on $\mathbb{M}$ is a section of the tangent bundle $T \mathbb{M}$. That means that, at each configuration $m \in \mathbb{M}, F$ is described by (and identified with) a linear map $\omega_{F}(m)$ from $T_{m} \mathbb{M}$ to $\mathbb{R}$ such that $m \mapsto \omega_{F}(m)$ is $\mathcal{C}^{\infty} \cdot \omega_{F}$ is then a section of the cotangent bundle $T^{*} \mathbb{M}$. It is also an 1-form or an element $\Lambda^{1}(\mathbb{M})$ because each linear form may be viewed as an skew-symmetric linear form! The set of vector fields on $\mathbb{M}$ is denoted by $\mathfrak{X}(\mathbb{M})$. It is then the set of sections of $T \mathbb{M}$. The set of sections of $T^{*} \mathbb{M}$ is denoted by $\mathfrak{X}^{*}(\mathbb{M})$. Both $\mathfrak{X}(\mathbb{M})$ and $\mathfrak{X}^{*}(\mathbb{M})$ are vector spaces.

Without any consideration of linearity, a major fact is that the set $\mathcal{F}$ of all the physical actions involved in $\Sigma$ is represented by (and identified with) the sum $\omega_{\mathcal{F}}=\sum_{F \in \mathcal{F}} \omega_{F} \in \mathfrak{X}^{*}(\mathbb{M})$ of all the sections of each individual physical action.

Definition 1. $\omega=\omega_{\mathcal{F}} \in \mathfrak{X}^{*}(\mathbb{M})$ is called the force system on $\mathbb{M}$.

In a chart $\left(U_{\alpha}, \psi_{\alpha}\right)$ we may write $\omega$ as:

$$
\omega(m)=\sum_{k=1}^{n} \omega_{k}(q) d q^{k} \quad \forall m \in U_{\alpha} \text { such that } \phi_{\alpha}(m)=q
$$

so that if $X \in \mathfrak{X}(\mathbb{M})$ is a vector field on the manifold $\mathbb{M}$, we may write $X(m)=\sum_{k=1}^{n} X^{k}(q) \frac{\partial}{\partial q^{k}}\left(\right.$ or $X(m)=\left(X^{1}(q), \ldots, X^{n}(q)\right)$ and we speak of coordinates of $X$ in the chart $\left(U_{\alpha}, \psi_{\alpha}\right)$ at $m$. Then

$$
\omega(X)(m)=\omega(m)(X(m))=\sum_{k=1}^{n} \omega_{k}(q) X^{k}(q)
$$

which does not depend on the choice of the chart at $m$. The family $\left(\omega_{k}(q)\right)_{k}$ are the coordinates of $\omega$ at $m$ in the chart $\left(U_{\alpha}, \psi_{\alpha}\right)$. In mechanics, when we apply the virtual power principle, we use any vector field $X$ and we note it $X^{*}$. Because we only need a basis of the vector fields, we only need and we only use the families $X_{k}^{*}\left(m^{\prime}\right)=(0, \ldots, 0,1,0, \ldots, 0)$ for $k=1, \ldots, n$ and for all $m^{\prime} \in U_{\alpha}$. Thus, that means emphasizing some directions $\left(\frac{\partial}{\partial q^{k}}\right.$ for $\left.k=1, \ldots, n\right)$ on the manifold, directions defined by the choice of the chart $\left(U_{\alpha}, \psi_{\alpha}\right)$. As far as possible, we will try to avoid such choice of charts and of preferential directions.

A lot of comments are necessary.

In the context of discrete mechanical systems, there is no distinction between boundary and volume actions. Such a distinction is crucial in continuum mechanics especially when a control is performed on the system. This control is frequently made only through the boundary of the system which leads to a special formulation of the evolution problem. [20] accurately investigates this issue in order to extend the second-order work criterion from material to boundary problem. The present paper then deals with boundary problems but the framework of discrete systems removes the distinction between boundary and volume actions. The force system $\mathcal{F}$ may be split in two parts according to two different classifications. The first classification distinguishes the external and the internal forces whereas the second one splits $\mathcal{F}$ into the given forces $\mathcal{F}_{g}$ and the link forces $\mathcal{F}_{\ell}$ so that $\mathcal{F}=\mathcal{F}_{g} \cup \mathcal{F}_{\ell}$. We adopt this second classification. By definition, the given forces are fully described by the kinematics of the 


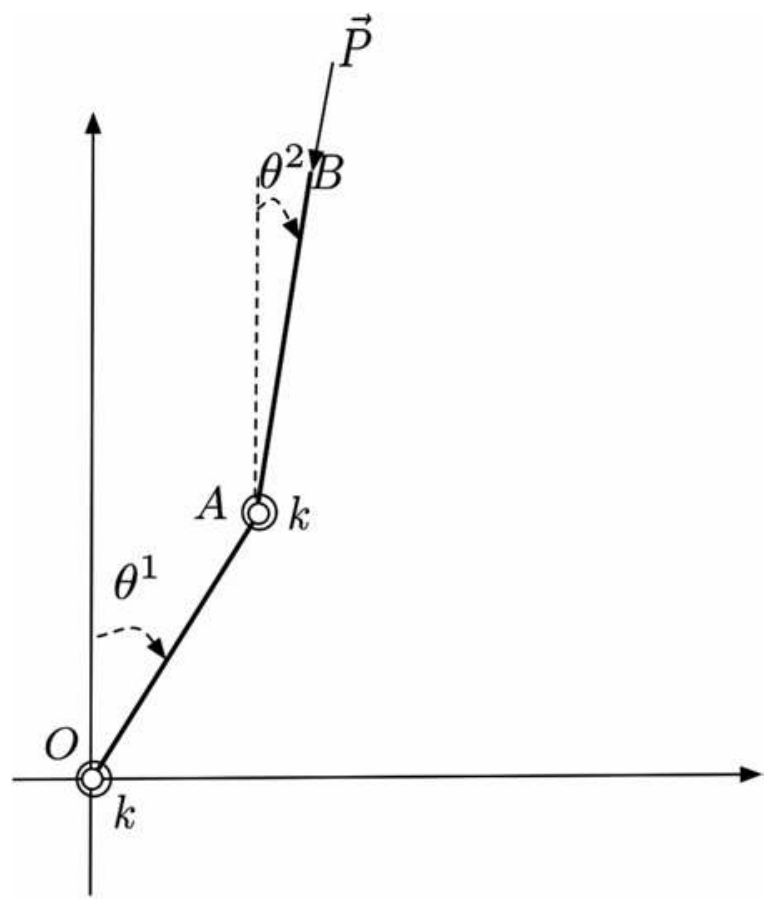

F I G U R E 1 Usual 2 d.o.f. Ziegler system with $\omega_{1}$ as force system

system. Because only positional forces are here taken into account, that means that the corresponding forces are given functions of $m \in \mathbb{M}$. Thus, it is represented by the section $\omega_{g}=\sum_{F \in \mathcal{F}_{g}} \omega_{F}$ of $T^{*} \mathbb{M}$.

In a laboratory and for experiments, a large part of these given actions are the control forces and another part may be for example the gravity force. The controlled part $\mathcal{F}_{L}$ of the given force system $\mathcal{F}_{g}$ leads to the

Definition 2. $\omega_{L}=\sum_{F \in \mathcal{F}_{L}} \omega_{F} \in \mathfrak{X}^{*}(\mathbb{M})$ is called the load system on $\mathbb{M}$.

The link forces are $\left\{\omega_{F} \in \mathfrak{X}^{*}(\mathbb{M}) \mid F \in \mathcal{F}_{\ell}\right\}$. This set may itself be split into two sets $\mathcal{F}_{\ell}=\mathcal{F}_{\ell, 1} \cup \mathcal{F}_{\ell, 2}$. For $F \in \mathcal{F}_{\ell, 2}$, $\omega_{F}\left(X^{*}\right)=0$ for any virtual velocity field $X^{*} \in \mathfrak{X}(\mathbb{M})$. That means that $\omega_{F}=0$ as element of $\mathfrak{X}^{*}(\mathbb{M})$ although the corresponding physical action $F$ does not vanish. In a laboratory and for experiments, a large part of the link forces $F \in \mathcal{F}_{\ell, 2}$ are forces associated by duality to the kinematic control of the system. This will be more clear when the concept of loading path will be later introduced and when submanifolds of $\mathbb{M}$ will be involved. The forces $F \in \mathcal{F}_{\ell, 2}$ are then removed in this formulation of the mechanic laws because they are orthogonal by duality to any virtual velocity fields $X^{*} \in \mathfrak{X}(\mathbb{M})$.

In the framework adopted in this paper, the constitutive laws of the links lead then to functions $m \mapsto \omega_{F}(m)$ for any $F \in \mathcal{F}_{\ell_{1}}$ and, for all $m \in \mathbb{M}, \omega_{\ell}(m)=\sum_{F \in \mathcal{F}_{\ell}} \omega_{F}(m)=\sum_{F \in \mathcal{F}_{\ell, 1}} \omega_{F}(m) \in T_{m}^{*} \mathbb{M}$ so that $\omega_{\ell} \in \mathfrak{X}^{*}(\mathbb{M})$. The fact that the section $\omega_{\ell}$ is a simple function of $m \in \mathbb{M}$ and does not depend of other variables means that the system is elastic. In this framework, a constitutive law of a link force is hyperelastic if there is a differentiable function $W_{F}$ on $\mathbb{M}$ such that $\omega_{F}=-d W_{F}$ where $d W_{F} \in \mathfrak{X}^{*}(\mathbb{M})$ is the differential of $W_{F}$. $W_{F}$ is called the elastic potential of $F$. A similar definition may be used for any given conservative action $F \in \mathcal{F}_{g}$ which has a potential energy $U_{F}: m \mapsto U_{F}(m)$ such that $\omega_{F}=-d U_{F}$. Note that, in this approach, the constitutive laws of the links given by the functions $m \mapsto \omega_{F}(m)$ for any $F \in \mathcal{F}_{\ell_{1}}$ are not incremental. These are full constitutive relations. We discuss later to the possible incremental description of these constitutive laws in a section devoted to elasticity.

To sum up, apart from eventual natural and permanent given actions like gravity, $\omega_{g}$ merges the so-called loading part of the force system whereas $\omega_{\ell}$ merges the constitutive part of the force system. The map $m \mapsto \omega_{\mathcal{F}}(m)=\omega(m)=\omega_{g}(m)+\omega_{\ell}(m)$ then summarizes all the actions that make $\omega_{\mathcal{F}} \in \mathfrak{X}^{*}(\mathbb{M})$ a section of $T^{*} \mathbb{M}$ and eliminates the unknowns forces involved in the kinematic control.

For example, in the usual 2 degree of freedom Ziegler systems $\Sigma=\left(S_{1}, S_{2}\right)$, the configuration set is the 2 dimensional torus $\mathbb{M}=\mathbb{S}^{1} \times \mathbb{S}^{1}$ with the coordinate system $q=\left(\theta^{1}, \theta^{2}\right)$ as on Figure 1. Each body $S_{i}$ is a bar of length $h$. The followed force $P$ is the unique given force and is also the unique element of the load system: $\mathcal{F}_{g}=\mathcal{F}_{L}=\{P\}$. It defines the section $\omega_{g}=\omega_{P}$ and calculations give:

$$
q=\left(\theta^{1}, \theta^{2}\right) \mapsto \omega_{g}(q)=\omega_{P}(q)=h P \sin \left(\theta^{1}-\theta^{2}\right) d \theta^{1}
$$




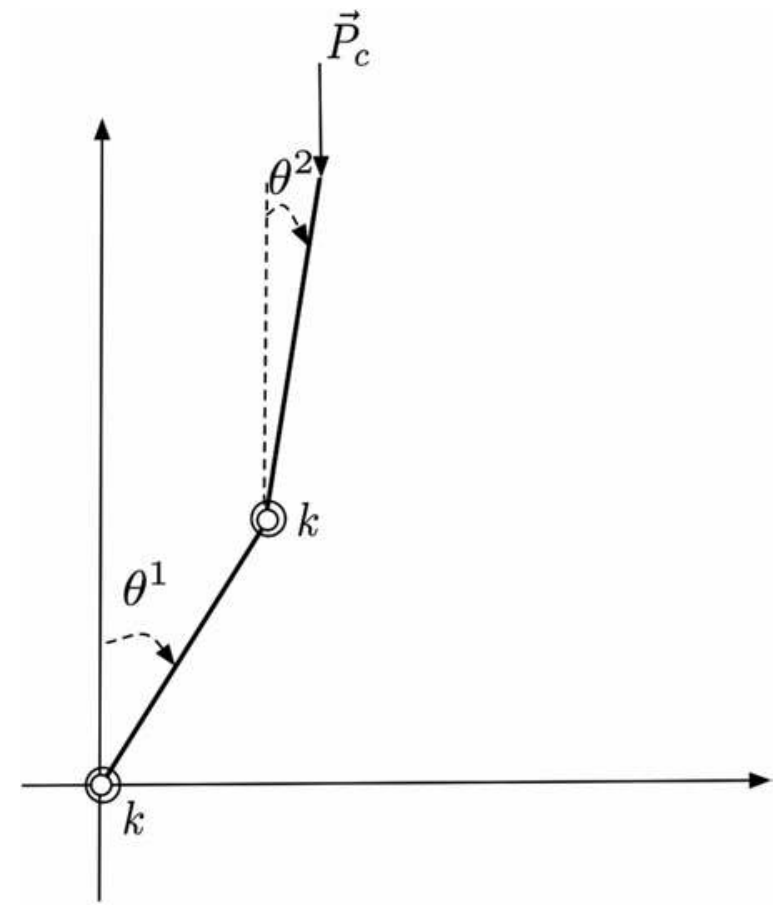

F I G U R E 2 Usual 2 d.o.f. conservative system with $\omega_{2}$ as force system

where, to simplify the presentation, we do not make the distinction between a point $m \in \mathbb{M}$ and its coordinates $q=\left(\theta^{1}, \theta^{2}\right)$ in the coordinate system.

The link forces involved in $\omega_{\ell}$ are $\mathcal{F}_{\ell, 1}=\left\{C_{1}, C_{12}\right\}$ where $C_{1} \vec{z}$ is the torque at $O$ of the action of the backing on $S_{1}$ and $C_{12} \vec{z}$ is the torque at $A$ of the action of $S_{1}$ on $S_{2}$ so that

$$
\omega_{\ell}=\left(C_{1}-C_{12}\right) d \theta^{1}+C_{12} d \theta^{2}
$$

thanks to the Action -reaction law. We adopt an elastic constitutive law with two same torsion stiffnesses $k$ at each joint. That means that $C_{1}=-k \theta^{1}$ and $C_{12}=-k\left(\theta^{2}-\theta^{1}\right)$. $\omega_{\ell}$ then reads:

$$
\omega_{\ell}(q)=\left(-2 k \theta^{1}+k \theta^{2}\right) d \theta^{1}-\left(k \theta^{2}-k \theta^{1}\right) d \theta^{2}
$$

$\omega_{\ell}(q)=-d W_{e}(q)$ with $W_{e}(m)=W_{e}(q)=\frac{1}{2}\left(2 k\left(\theta^{1}\right)^{2}+k\left(\theta^{2}\right)^{2}-2 k \theta^{1} \theta^{2}\right)$ in this local chart. The force system is $\mathcal{F}=$ $\left\{P, C_{1}, C_{12}\right\}$, the loading system is $\mathcal{F}_{g}=\{P\}$ and the corresponding section $\omega=\omega_{1}$ then reads in this coordinate system:

$$
\begin{aligned}
q=\left(\theta^{1}, \theta^{2}\right) \mapsto \omega_{1}\left(\theta^{1}, \theta^{2}\right) & =\left(-2 k \theta^{1}+k \theta^{2}+h P \sin \left(\theta^{1}-\theta^{2}\right)\right) d \theta^{1}+\left(k \theta^{1}-k \theta^{2}\right) d \theta^{2} \\
& =\omega_{1,1}\left(\theta^{1}, \theta^{2}\right) d \theta^{1}+\omega_{1,2}\left(\theta^{1}, \theta^{2}\right) d \theta^{2}
\end{aligned}
$$

$\left(\omega_{1,1}, \omega_{1,2}\right)$ are the coordinates of $\omega_{1}$ in the local chart $\left(d \theta^{1}, d \theta^{2}\right)$ associated to the local chart $q=\left(\theta^{1}, \theta^{2}\right)$ of $\mathbb{M}$. It is well known that the only equilibrium position for such a loading system $\mathcal{F}_{L}=\mathcal{F}_{g}=\{P\}$ is the point $(0,0)$ for any $P>0$.

Consider now the analogous conservative system with a dead load $P_{c}$ at the extremity as on Figure 2 . The corresponding section $\omega_{2}$ then reads:

$$
\begin{aligned}
q=\left(\theta^{1}, \theta^{2}\right) \mapsto \omega_{2}\left(\theta^{1}, \theta^{2}\right) & =\left(-2 k \theta^{1}+k \theta^{2}+h P_{c} \sin \theta^{1}\right) d \theta^{1}+\left(k \theta^{1}-k \theta^{2}+h P_{c} \sin \theta^{2}\right) d \theta^{2} \\
& =\omega_{2,1}\left(\theta^{1}, \theta^{2}\right) d \theta^{1}+\omega_{2,2}\left(\theta^{1}, \theta^{2}\right) d \theta^{2}
\end{aligned}
$$

$\left(\omega_{2,1}, \omega_{2,2}\right)$ are the coordinates of $\omega_{2}$ in the local chart $\left(d \theta^{1}, d \theta^{2}\right)$ associated to the local chart $q=\left(\theta^{1}, \theta^{2}\right)$ of $\mathbb{M}$. Contrary to the above 2 dof Ziegler system, according to the values of $P_{c}$, there are different equilibrium configurations. We discuss below this issue. 


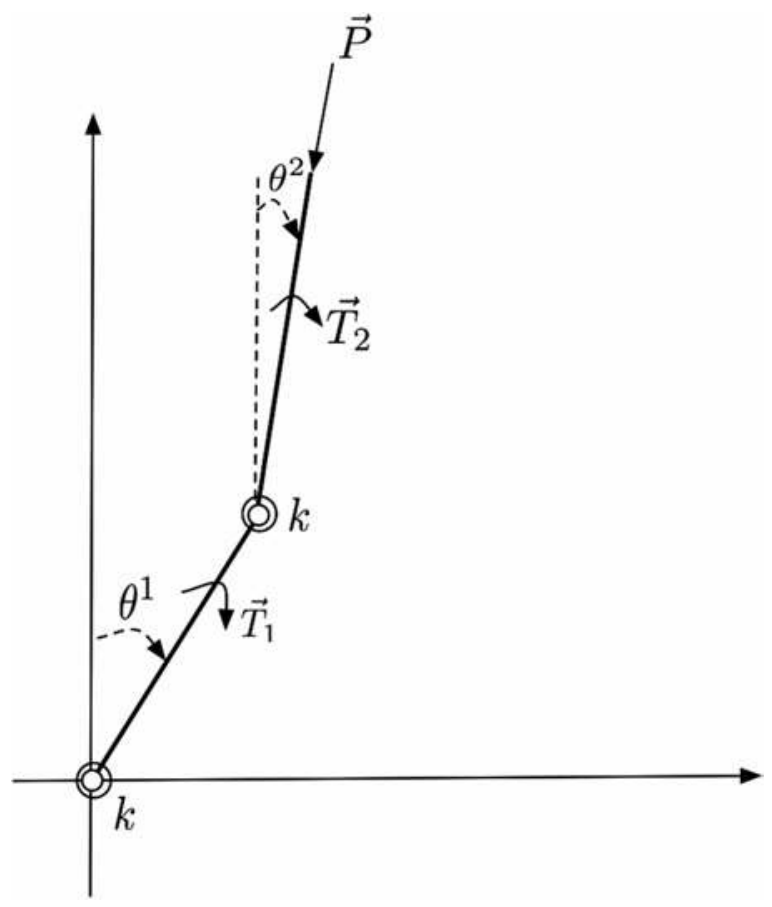

F I G U R E 3 Usual 2 d.o.f. Ziegler system with $\omega_{3}$ as force system

In order to consider an infinite sequence of different equilibriums for the Ziegler system, we then must consider other load systems than $\omega_{P}$ which leads to only one equilibrium. For example, we may envisage direct external torques $\vec{T}_{i}=T_{i} \vec{z}$ on $S_{i}$ (see Figure 3) so that for the load system $\mathcal{F}_{L}=\mathcal{F}_{g}=\left\{P, T_{1}, T_{2}\right\}$, the corresponding section $\omega_{3}$ reads:

$$
\begin{aligned}
\omega_{3}(q)=\omega_{3}\left(\theta^{1}, \theta^{2}\right) & =\left(-2 k \theta^{1}+k \theta^{2}+h P \sin \left(\theta^{1}-\theta^{2}\right)+T_{1}\right) d \theta^{1}+\left(k \theta^{1}-k \theta^{2}+T_{2}\right) d \theta^{2} \\
& =\omega_{3,1}\left(\theta^{1}, \theta^{2}\right) d \theta^{1}+\omega_{3,2}\left(\theta^{1}, \theta^{2}\right) d \theta^{2}
\end{aligned}
$$

$\left(\omega_{3,1}, \omega_{3,2}\right)$ are the coordinates of $\omega_{3}$ in the local chart $\left(d \theta^{1}, d \theta^{2}\right)$ associated to the local chart $q=\left(\theta^{1}, \theta^{2}\right)$ of $\mathbb{M}$.

\section{2 | VECTOR BUNDLES}

There are numerous references about vector bundles (see for example [3,21] and [8] or [4] for francophone people). We already met two usual vector bundles 'over' $\mathbb{M}$ : the tangent bundle $T \mathbb{M}$ and the cotangent bundle $T^{*} \mathbb{M}$ that encode two fundamental objects of mechanics: the infinitesimal displacements and the virtual/real velocity fields for the former one and the force systems for the latter one.

Generally, a vector bundle $E$ over a manifold $B$ is the given of a $C^{\infty}$ manifold structure on $E$, a surjective differentiable map $\pi: E \rightarrow B$ (in fact a submersion) called the projection of the bundle such that, locally over open sets $U$ of $B$ called the charts of the fiber bundle, $\pi^{-1}(U)$ is diffeomorphic to $U \times F$ where $F$ is a given vector space (local triviality property of $E$ ). If $x \in B$ then $E_{x}=\pi^{-1}\{x\}$ is a vector space isomorphic to $F$ and is called the fiber over $x$. If $\operatorname{dim} F=r$ and $\operatorname{dim} B=n$ then $E$ is a $n+r$ manifold. For $E=T \mathbb{M}, F=\mathbb{R}^{n}$ and $B=\mathbb{M}, T \mathbb{M}_{m}=T_{m} \mathbb{M}$ is the tangent space of $\mathbb{M}$ at $m$. For $T^{*} \mathbb{M}, F=\left(\mathbb{R}^{n}\right)^{*}$ the dual space of $\mathbb{R}^{n}$ and $B=\mathbb{M}, T^{*} \mathbb{M}_{m}=T_{m}^{*} \mathbb{M}$ is the cotangent space of $\mathbb{M}$ at $m \cdot \operatorname{dim} T \mathbb{M}=\operatorname{dim} T^{*} \mathbb{M}=2 n$.

The objects naturally associated to a vector bundle $E$ over $B$ are the so-called sections of $E$. A section $s$ of $E$ is a differentiable map $s: B \rightarrow E$ such that $\pi \circ s=i d_{B}$. The set of sections of $E$ is denoted by $\Gamma(E)$. Because of the local triviality property of $E$, there are always local sections. In vector bundles which are special cases of more general fiber bundles, there is a special section which is a global section: the nil section denoted by $0_{E}$ or 0 if no confusion is possible. $\Gamma(E)$ is a vector space with $0_{E}$ as nil vector. Even if a section $s$ is a map, then, like for real functions, we draw a section $s$ by its image in $E$. The nil section $0_{E}$ is a submanifold of $E$ canonically diffeomorphic to $B$ and often identified with $B$ that makes $B$ as a submanifold of $E$.

We only remark that the existence of a not nil global section is a deep question whose answer gives significant informations on the nature of the topology of $B$. It is out of the scope of the paper and refers to the so-called global issues on $B$. 


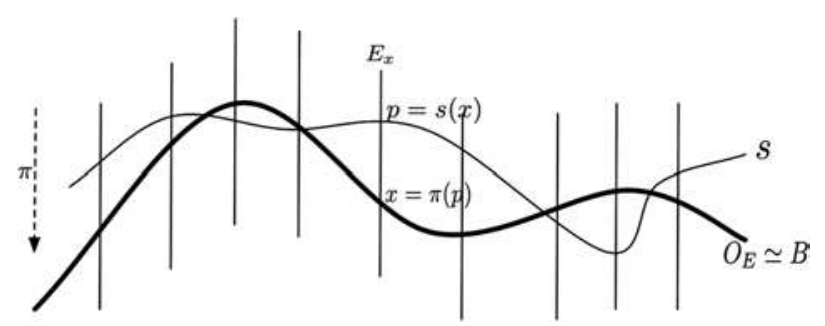

F I G U R E 4 Vector bundle

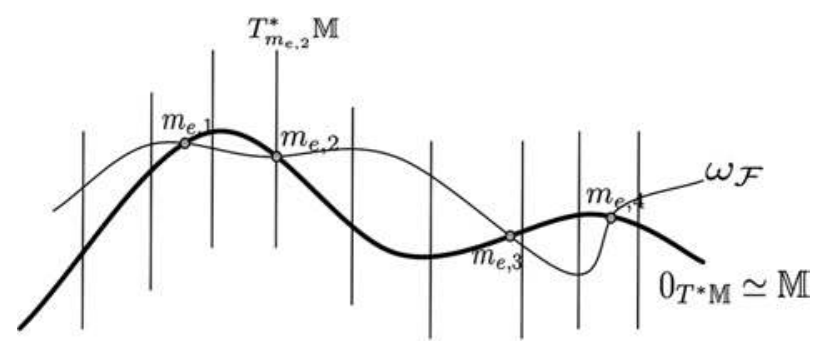

F I G URE 5 Equilibrium

The following picture (4) sums up the above concepts. Be aware that this picture is drawn with $n=1$ and $r=1$. With $n=2$ and for tangent and cotangent bundles, the picture should necessitate 4 dimensions and is not drawable.

\section{3 | EQUILIBRIUM AND STABILITY}

Definition 3. A configuration $m_{e}$ under a force system $\omega_{\mathcal{F}}$ is an equilibrium configuration if $\omega_{\mathcal{F}}\left(m_{e}\right)=0=0_{T^{*} \mathbb{M}}\left(m_{e}\right)$. Referring to a coordinate system $\left(q_{k}\right)_{k}$ and using (1), it is equivalent to a family of $n$ equations $\omega_{\mathcal{F}, k}(q)=0 \quad \forall k=1, \ldots, n$ that are the so-called equilibrium equations. They are generally strongly non linear in the coordinate system $\left(q_{k}\right)_{k}$. Because $\omega_{\mathcal{F}}$ is a section of the vector bundle $T^{*} \mathbb{M}$, this geometrically means that the section $\omega_{\mathcal{F}}$ cuts or intersects the nil section at $m_{e}$.

On the picture (5), the force system $\mathcal{F}$ has four equilibrium positions $m_{e, 1}, m_{e, 2}, m_{e, 3}, m_{e, 4}$.

The previous definition provides the geometric meaning of an equilibrium position $m_{e}$. We now focus on the geometrical meaning of the stability of $m_{e}$ for a force system $\mathcal{F}$ namely for $\omega_{\mathcal{F}}$.

In the static framework, there are two possible ways to define the stability. The first one refers to the infinitesimal unicity of the equilibrium configuration: $m_{e}$ is stable for the considered force system $\mathcal{F}$ if there is no other equilibrium infinitely close to $m_{e}$ (see for example [18]). The second one uses infinitesimal perturbations of the force system: $m_{e}$ is stable for the considered force system $\mathcal{F}$ if an infinitesimal perturbation $\delta \mathcal{F}$ of the force system $\mathcal{F}$ leads to an equilibrium $m_{e}^{\prime}$ infinitely close to $m_{e}$. The infinitesimal perturbation $\delta \mathcal{F}$ is realized by an infinitesimal perturbation $\delta \mathcal{F}_{L}$ of the load system $\mathcal{F}_{L} \subset \mathcal{F}_{g} \subset \mathcal{F}$. The corresponding section $\omega_{\mathcal{F}}+\omega_{\delta L}$ must then cut the nil section of $T^{*} \mathbb{M}$ at $m_{e}^{\prime}$ infinitely close to $m_{e}$. These two conditions are in fact equivalent. Their geometric translation leads to the following definition of the stability. In order to be as clear as possible, we decide to introduce the specific term of Transversality-stability or T-stability of $m_{e}$.

Definition 4. Let $\mathcal{F}$ be a force system described by a section $\omega_{\mathcal{F}}$ of $T^{*} \mathbb{M}$ and $m_{e} \in \mathbb{M}$ an equilibrium configuration of $\Sigma$ subjected to $\mathcal{F}$. Thus $\omega_{\mathcal{F}}\left(m_{e}\right)=0=0_{T^{*} \mathbb{M}}\left(m_{e}\right) . m_{e}$ is called Transervality-stable or T-stable if $\omega_{\mathcal{F}}$ intersects or cuts transversally the nil section $0_{T * \mathbb{M}}$.

On the following Figure 6, a small perturbation $\delta \mathcal{F}=\delta \mathcal{F}_{L}$ of the force system $\mathcal{F}$ leads to two new equilibrium configurations $m_{e, 1}^{\prime}$ and $m_{e, 2}^{\prime}$ close respectively to $m_{e, 1}$ and $m_{e, 2}$ but not for $m_{e, 3}: m_{e, 1}, m_{e, 2}$ are T-stable equilibriums whereas $m_{e, 3}$ is an T-unstable equilibrium.

This illustrates the geometric meaning of the T-stability of equilibriums without referring to any regularity of the so-called tangent stiffness matrices. This criterion is purely geometric, non-linear and involves the mode of intersections of two submanifolds of a manifold according to the following 


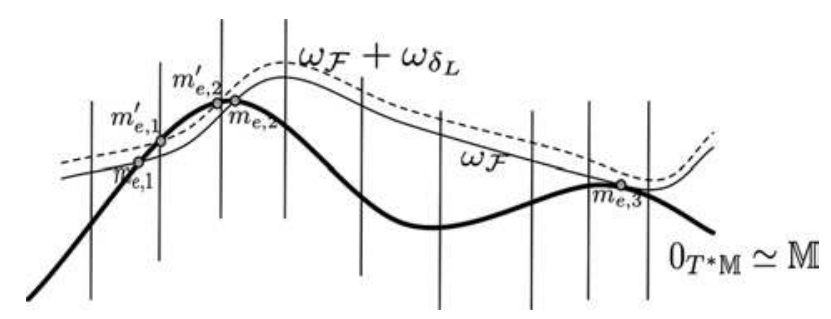

F I G U R E 6 T-stable $\left(m_{e, 1}, m_{e, 2}\right)$ and T-unstable $m_{e, 3}$ equilibriums

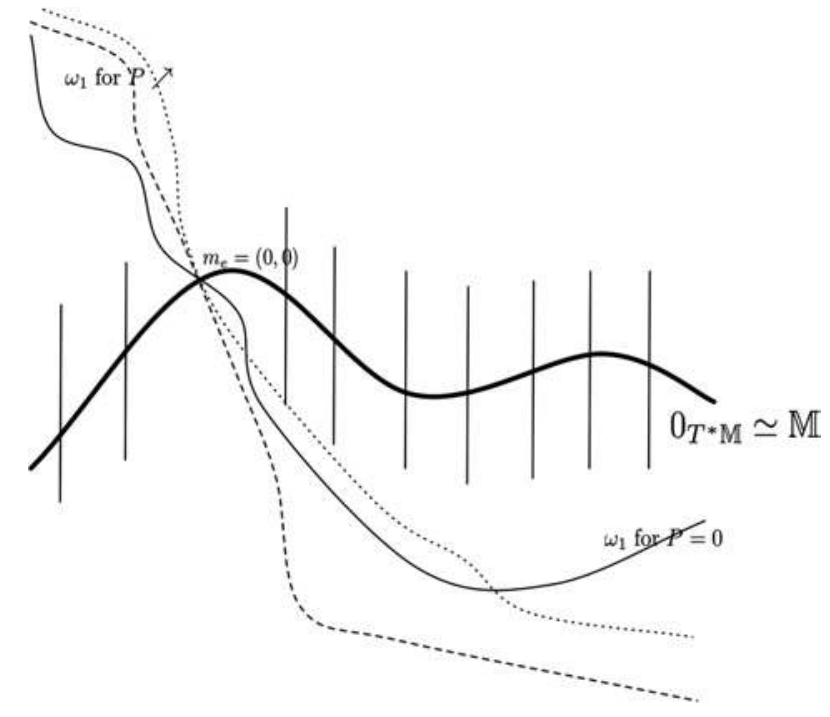

F I G U R E 7 T-stability of $m_{e}=(0,0)$ of the Ziegler system for any $P$

Definition 5. Two submanifolds $V$ and $W$ of a manifold $P$ are transverse at $p \in V \cap W$ if and only if $T_{p} V+T_{p} W=T_{p} P$. More generally if $f: N \rightarrow P$ is a $C^{\infty}$ map, $W$ is a submanifold of $P$ and $x \in N$ with $f(x)=p \in W$. $f$ is transverse to $W$ at $p\left(\right.$ in $P$ ) if $\operatorname{Imd} f(x)+T_{f(x)} W=T_{p} P$.

The T-stability of $m_{e}$ is then equivalent to the transversality of $\omega_{\mathcal{F}}$ to $0_{T^{*} \mathbb{M}}(\mathbb{M}) \simeq \mathbb{M}$ in $T^{*} \mathbb{M}$ at $m_{e}$. But, referring to the above definition, this actually involves the derivative of the sections which is the crux of the problem. Indeed, as mentioned in the introduction, making derivatives of sections of a vector bundle $E$ necessitates a connection on $E$ whereas no natural connection emerges from the mechanical problem. We will then avoid the difficulty by using the existence of the global intrinsic nil section $0_{E}$ in a vector bundle.

To conclude this section devoted to geometrical features, we now translate the T- stability of the equilibrium position $m_{e}=$ $(0,0)$ with this language for both the Ziegler system (Figure 1) and the conservative system (Figure 2). Figure 7 refers to the Ziegler system with the load $P$ increasing from 0 . When the follower nonconservative load $P$ is increasing, the above section $\omega_{1}$ (Equation 2) is changing but it always keeps transversal to the nil section at $m_{e}=(0,0)$ and only at $m_{e}=(0,0)$. This means that $m_{e}=(0,0)$ is the unique equilibrium and is always T-stable. On the contrary, on Figure 8 is drawn the evolution of the above section $\omega_{2}$ (Equation 3) for a vertical conservative loading $P_{c}$ increasing from 0 . When $P_{c}$ reaches the critical value $P_{c}^{*}=$ $(3-\sqrt{5}) \frac{k}{h}$, the equilibrium configuration $m_{e}=(0,0)$ ceases to be T-stable whereas another T-stable equilibrium configuration $m_{e}^{\prime}$ may rise: the T-instability is then associated with a bifurcation. We take the opportunity of this example to stress that the definition of T-stability cannot describe by itself the post-critical behavior nor the variety of possible bifurcations.

We now tackle the infinitesimal aspect and we deduce the link between T-stability and divergence stability.

\section{4 | INFINITESIMAL ASPECTS}

Infinitesimal or tangent aspects of the question involve the tangent bundle $T E$ of $E$ namely $T T^{*} \mathbb{M}$. In $T E$ there is a natural subbundle $V E$ called the vertical bundle consisting in the collection, at each $p \in E$ of the vectors of $E_{m}$ where $m=\pi(p)$. 


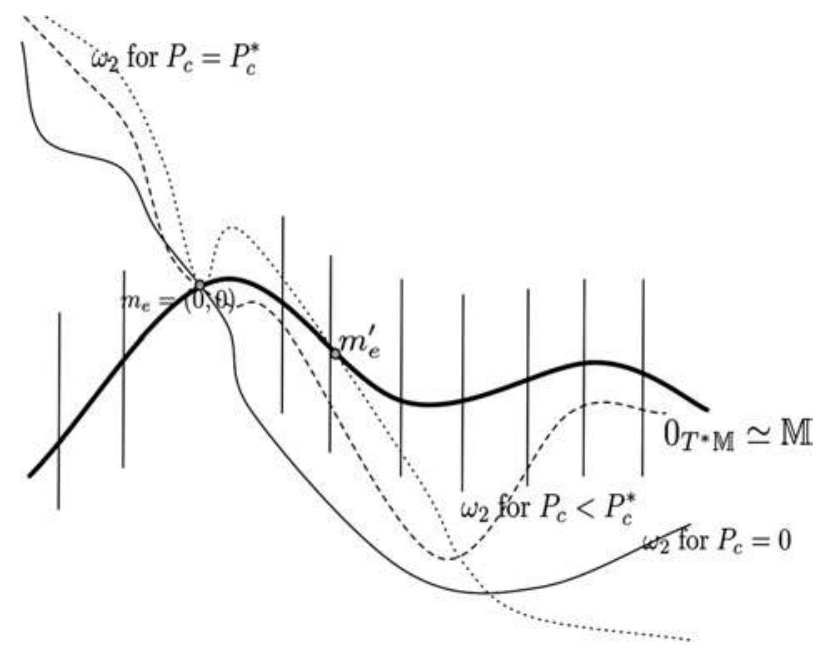

F I G U R E 8 T-stability of $m_{e}=(0,0)$ of the associated conservative system for $P_{c}<P_{c}^{*}$ and T-instability for $P_{c}=P_{c}^{*}$

This is performing because the tangent space of any vector space at any point can be identified with the vector space itself. Unfortunately there is no natural way to have a supplementary $H E$ of $V E$ in $T E$. In fact, giving a smooth distribution $p \mapsto H_{p} E$ of supplementary spaces of $V_{p} E$ in $T_{p} E$ is equivalent to give a connection or a covariant derivative $\nabla$. This fact is then exactly the connection issue which we would like to avoid.

Though we plan to investigate an evolution on the section $0_{E} \simeq B$ namely a quasi-static evolution of $\Sigma$. Let $s \in \Gamma(E)$ be any section of $E$. Let $x_{e} \in B$ such that $s\left(x_{e}\right)=\left(x_{e}, 0\right)$. First there is for any such $x \in B$ a canonical decomposition

$$
T_{(x, 0)} E \simeq T_{x} B \oplus E_{x}
$$

This decomposition induces at $x_{e}$ the following form for the differential $d s$ of $s$ at $x_{e}$ :

$$
\begin{aligned}
d s\left(x_{e}\right): T_{x_{e}} B & \rightarrow T_{\left(x_{e}, 0\right)} E=T_{x_{e}} B \oplus E_{x_{e}} \\
u & \mapsto u+d s^{v e r}\left(x_{e}\right)(u)
\end{aligned}
$$

where $d s^{v e r}\left(x_{e}\right)$ is a linear map and then belongs to $\mathcal{L}\left(T_{x_{e}} B, E_{x_{e}}\right)$. ( $d s$ must not be confused with the exterior derivative $\mathbf{d} s$ when $s=\omega$ is an 1 -form).

Then the transversality condition of $s$ at $x_{e}$ with the nil section means that $d s^{v e r}\left(x_{e}\right)$ is a surjective map and then

$$
T_{\left(x_{e}, 0\right)} E=T_{x_{e}} B+\operatorname{Im}\left(d s^{v e r}\left(x_{e}\right)\right)
$$

When $s$ intersects $0_{E}$ transversally at $x_{e}$, we write $s \top^{x_{e}} 0_{E}$.

We now apply these general results to the cotangent bundle $T^{*} \mathbb{M}$ of $\mathbb{M}$. Let $m_{e}$ be an equilibrium configuration of a mechanical system $\Sigma$ subjected to the force system $\mathcal{F}$.

Definition 6. The above linear map $d \omega_{\mathcal{F}}^{\text {ver }}\left(m_{e}\right) \in \mathcal{L}\left(T_{m_{e}} \mathbb{M}, T_{m_{e}}^{*} \mathbb{M}\right)$ is called the tangent stiffness operator or the tangent stiffness tensor of $\Sigma$ at $m_{e}$. Because of the involved spaces, it is a 2 order covariant tensor on the vector space $T_{m_{e}} \mathbb{M}$. It obviously depends on $m_{e}$ and on the force system $\mathcal{F}$.

$T_{m_{e}} \mathbb{M}$ and $T_{m_{e}}^{*} \mathbb{M}$ have the same dimension. Thus, thanks to the rank theorem, the transversality condition means that $d \omega_{\mathcal{F}}^{v e r}\left(m_{e}\right)$ is a bijective map. We then deduce now as a theorem and not as a definition the infinitesimal T-stability condition for the equilibrium $m_{e}$ :

Proposition 1. $m_{e}$ is T-stable if and only if $d \omega_{\mathcal{F}}^{v e r}\left(m_{e}\right)$ is an invertible map.

The matrix $K\left(q_{e}\right)$ of $d \omega_{\mathcal{F}}^{v e r}\left(m_{e}\right)$ in the natural bases $\left(e_{i}=\left.\frac{\partial}{\partial q^{i}}\right|_{m_{e}}\right)_{i}$ of $T_{m_{e}} \mathbb{M}$ and its dual basis for $T_{m_{e}}^{*} \mathbb{M}$ is the so-called tangent stiffness matrix of the system at $q_{e}$ which is the coordinates of $m_{e}$ in the local chart $q$. The divergence stability criterion reads $\operatorname{det} K\left(q_{e}\right) \neq 0$ and claims the invertibility of the matrix $K\left(q_{e}\right)$. Divergence stability is then equivalent to T-stability. From now on, we consider $d \omega_{\mathcal{F}}^{v e r}\left(m_{e}\right)$ as a $(0,2)$-tensor. 
As any $(0,2)$ tensor, $d \omega_{\mathcal{F}}^{\text {ver }}\left(m_{e}\right)$ naturally splits into its symmetric $d \omega_{\mathcal{F}}^{\text {ver,s }}\left(m_{e}\right)$ and skew-symmetric $d \omega_{\mathcal{F}}^{\text {ver,a }}\left(m_{e}\right)$ parts. Then

Theorem 2.

$$
\mathbf{d} \omega_{\mathcal{F}}\left(m_{e}\right)=d \omega_{\mathcal{F}}^{v e r, a}\left(m_{e}\right)
$$

Hill's second order work criterion involves the symmetric part $d \omega_{\mathcal{F}}^{\text {ver,s }}\left(m_{e}\right)$ :

Definition 7. $m_{e}$ is Hill-stable for a force system $\mathcal{F}$ if $d \omega_{\mathcal{F}}^{v e r, s}\left(m_{e}\right)$ is positive definite.

Then,

Proposition 3. If $m_{e}$ is $T$-unstable for a force system $\mathcal{F}$ then it is Hill-unstable for the same force system $\mathcal{F}$.

Proof. If $m_{e}$ is T-unstable, then $d \omega_{\mathcal{F}}^{v e r}\left(m_{e}\right)$ is not invertible. Thus it is not an injective map and here is a vector $U \in T_{m_{e}} \mathbb{M}, U \neq 0$ such that $d \omega_{\mathcal{F}}^{v e r}\left(m_{e}\right)(U)=0$ namely $d \omega_{\mathcal{F}}^{v e r}\left(m_{e}\right)(U, V)=0 \quad \forall V \in T_{m_{e}} \mathbb{M}$ when it is viewed as a $(0,2)$-tensor. We deduce that $d \omega_{\mathcal{F}}^{\text {ver }}\left(m_{e}\right)(U, U)=0$ but $d \omega_{\mathcal{F}}^{\text {ver }}\left(m_{e}\right)(U, U)=d \omega_{\mathcal{F}}^{\text {ver,s }}\left(m_{e}\right)(U, U)$ and $d \omega_{\mathcal{F}}^{\text {ver,s }}\left(m_{e}\right)$ is not positive definite: $m_{e}$ is not Hill-stable. Let us remark that the T-stability is a surjective property whereas the link with the Hill-stability is made thanks to an injectivity property by using the equivalence in finite dimension between injectivity and surjectivity for linear maps between two vector spaces of same dimension.

Before focusing on variable loading systems, let us make the computations on the 2 dof Ziegler system with the above loading system $L=\left\{P, T_{1}, T_{2}\right\} \subset \mathcal{F}$.

According to the expression 4 of $\omega_{3}$, the (unique) equilibrium configuration $q_{e}=\theta_{e}=\left(\theta_{e}^{1}, \theta_{e}^{2}\right)$ (still the intentional confusion of $m_{e}$ and its coordinates $q_{e}$ to simplify the notations) is solution of:

$$
\left\{\begin{array}{l}
\omega_{3,1}\left(\theta^{1}, \theta^{2}\right)=-2 k \theta^{1}+k \theta^{2}+h P \sin \left(\theta^{1}-\theta^{2}\right)+T_{1}=0 \\
\omega_{3,2}\left(\theta^{1}, \theta^{2}\right)=-k \theta^{2}+k \theta^{1}+T_{2}=0
\end{array}\right.
$$

whose solution is $q_{e}=\theta_{e}=\left(\theta_{e}^{1}, \theta_{e}^{2}\right)$ given by

$$
\left\{\begin{array}{l}
\theta_{e}^{1}=\frac{1}{k}\left(T_{1}+T_{2}-h P \sin \left(\frac{T_{2}}{k}\right)\right) \\
\theta_{e}^{2}=\frac{1}{k}\left(T_{1}+2 T_{2}-h P \sin \left(\frac{T_{2}}{k}\right)\right)
\end{array}\right.
$$

For $\left.\left.U=\left.u^{1} \frac{\partial}{\partial \theta^{1}}\right|_{m_{e}}\right)+\left.u^{2} \frac{\partial}{\partial \theta^{2}}\right|_{m_{e}}\right)=u^{1} e_{1}+u^{2} e_{2}$ and $V=v^{1} e_{1}+v^{2} e_{2}$ two any vectors of $T_{m_{e}} \mathbb{M}$ :

$$
d \omega_{3}^{v e r}\left(m_{e}\right)(U, V)=\left(2 k-h P \cos \left(\frac{T_{2}}{k}\right)\right) u^{1} v^{1}+h P \cos \left(\frac{T_{2}}{k}\right) v^{1} u^{2}-k u^{1} v^{2}+k u^{2} v^{2}
$$

or equivalently

$$
d \omega_{3}^{v e r}\left(m_{e}\right)=\left(2 k-h P \cos \left(\frac{T_{2}}{k}\right)\right) d \theta^{1} \otimes d \theta^{1}+h P \cos \left(\frac{T_{2}}{k}\right) d \theta^{2} \otimes d \theta^{1}-k d \theta^{1} \otimes d \theta^{2}+k d \theta^{2} \otimes d \theta^{2}
$$

Before tackling the loading paths, we now briefly discuss the general framework of elasticity.

\section{5 | ELASTICITY, HYPERELASTICITY, HYPOELASTICITY AND INCREMENTAL HYPOELASTICITY}

In this section, we then briefly revisit in the vector bundle language the meaning of elasticity and hypo-elasticity as constitutive laws of materials and systems. The literature is wide and rich on this topic. We may refer for example to basic papers like. ${ }^{[1,24,25]}$ The goal of this section is only to show how translating these concepts into the geometric language especially because it is the framework of the constitutive laws used in the following sections. In fact, a forthcoming paper will be entirely devoted to the topic of the response of the systems in the larger framework of hypo-elasticity and plasticity. We put the following 


\section{Definition 8.}

1. A mechanical elastic system is a couple $\left(\mathbb{M}, \omega_{e}\right)$ where $\mathbb{M}$ is a manifold (the configuration manifold) and $\omega_{e} \in \mathfrak{X}^{*}(\mathbb{M})$ is a section of the cotangent bundle $T^{*} \mathbb{M}$.

2. An elastic system $\left(\mathbb{M}, \omega_{e}\right)$ is hyperelastic if there is a function $W_{e}: \mathbb{M} \mapsto \mathbb{R}$ such that $\omega_{e}=-d W_{e}$.

3. An elastic system ( $\left.\mathbb{M}, \omega_{e}\right)$ is (weak) hypoelastic if $\mathbf{d} \omega_{e} \neq 0$ (exterior derivative).

There is a stronger hypoelasticity which is not globally defined by a section of $\omega_{e}$ but only by its infinitesimal tangent aspects (rate formulation):

Definition 9. A mechanical incremental hypoelastic constitutive law is a n dimensional differential system $\omega \mapsto \tau_{e}(\omega)$ on $T^{*} \mathbb{M}$ transverse to the vertical bundle $V\left(T^{*} \mathbb{M}\right)$ namely such that $T_{\omega} T^{*} \mathbb{M}=\tau_{e}(\omega) \oplus T_{\omega} \pi^{-1}\{\pi(\omega)\}=\tau_{e}(\omega) \oplus V(\omega)$ for all $\omega \in T^{*} \mathbb{M}$.

This definition needs some additional remarks.

- If this differential system on $T^{*} \mathbb{M}$ is integrable, then a section $\omega_{e}: m \mapsto \omega_{e}(m)$ of $T^{*} \mathbb{M}$ is "canonically" deduced by the following way. In an elastic system, there is a special so-called released configuration we denote by $m_{0}$ such that the internal forces are nil at this configuration. Then the section $\omega_{e}$ is the solution of the integrable system $\tau_{e}$ such that $\omega_{e}\left(m_{0}\right)=0$ namely the integral manifold of $\tau_{e}$ passing by $\left(m_{0}, 0\right) \in T^{*} \mathbb{M}$.

- In fact, because constitutive laws must be invariant under global euclidean displacement of the system, the corresponding section $\omega_{e}$ or the corresponding differential system $\tau_{e}$ must satisfy some specific properties. The group of euclidean displacements $\mathbb{D}$ is a Lie group acting on $\mathbb{M}$ and this action extends to $T^{*} \mathbb{M}$. The section is then equivariant under this action. This point is not the central aim of this paper and it will be more deeply investigated in a forthcoming paper.

- In the four grains example (see Section 8), the distribution is not integrable. It would be interesting to investigate the integrability conditions. It will be also more deeply investigated in a forthcoming paper.

Suppose now that the system is hyperelastic defined by $\left(\omega_{\ell}=\omega_{e}=-d W_{e}\right)$ and suppose also that the load $L$ also has a potential energy $U_{L}$. Let $W=U_{L}+W_{e}$ be the total potential energy and let $m_{e}$ be an equilibrium configuration when $\Sigma$ undergoes the given load $L: \omega\left(m_{e}\right)=-d W\left(m_{e}\right)=0$. Then $d \omega^{\text {ver }}\left(m_{e}\right)=d \omega^{\text {ver, },}\left(m_{e}\right)=-H_{W}\left(m_{e}\right)$ where $H_{W}\left(m_{e}\right)$ is the Hessian of $W$ at $m_{e}$ which is well-defined as a quadratic form on $T_{m_{e}} \mathbb{M}$. Then, according to the above definition, $m_{e}$ is T-stable if $H_{W}\left(m_{e}\right)$ is a non degenerate quadratic form. We have to stress that this condition is NOT the traditional condition which reads $H_{W}\left(m_{e}\right)$ positive definite and which corresponds, in this symmetric case, to the above Hill-stability condition. This later condition is a stronger condition which provides, in this case, the dynamic (Lyapounov) stability of the corresponding equilibrium $m_{e}$. However, as usual, if $H_{W}=H_{W}(p)$ continuously depends on an increasing (from 0) parameter $p$ and if $H_{W}(0)\left(m_{e}\right)$ is positive definite, then the critical parameter $p^{*}>0$ making $H_{W}\left(p^{*}\right)\left(m_{e}\right)$ degenerate (loss of T-stability) is also the critical value of $p$ making $H_{W}(p)\left(m_{e}\right)$ no longer positive definite (usual loss of stability). This is illustrated on Figure 8 when $p=P_{c}$ and $m_{e}=(0,0)$ in the used local chart.

From now on, except for the example of the diamond pattern in Section 8, all the systems will be supposed elastic $\left(\omega_{\ell}=\omega_{e}\right)$. We focus on the other part of the force system $\omega_{\mathcal{F}}$. We have in hands the tools to tackle quasi static evolutions under loading paths. The most general situation where loading paths involve systems with only incremental constitutive laws is reported to a forthcoming paper devoted more generally to plasticity.

\section{I LOADING PATHS}

We recall that a load system is a subset $\mathcal{F}_{L} \subset \mathcal{F}_{g} \subset \mathcal{F}$ of a force system described by a section $\omega_{L}$ of the cotangent bundle $T^{*} \mathbb{M}$. Then, $\omega=\omega_{F}=\omega_{L}+\omega_{G}+\omega_{\ell}=\omega_{g}-\omega_{R}$ where $\omega_{g}=\omega_{L}+\omega_{G}$ collects all the given permanent actions $\omega_{G}$ like gravity and the proper loading of the system $\omega_{L}$ whereas $\omega_{R}=-\omega_{\ell}$ is the section describing the response of the system. Elasticity $\left(\omega_{\ell}=\omega_{e}\right)$ means that this section $\omega_{R}=-\omega_{e}$ is given, invariable during the loading path. Only the part $\omega_{L}$ may be variable because the forces $F \in \mathcal{F}_{L}$ are the control forces. We now define a loading path as it is concretely used in experiences for example in geomechanics.

Adopting a Lagrangian approach (in contrast to the Newton one and not to the Eulerian one) that favours the kinematics over the forces, a loading path $\mathcal{L}$ is first the choice of an (embedded) submanifold $\mathbb{V}_{\mathcal{L}}$ of $\mathbb{M}$ such that the configurations of $\Sigma$ along the loading path are on $\mathbb{V}_{\mathcal{L}}$ : this describes the kinematic part of the control. 


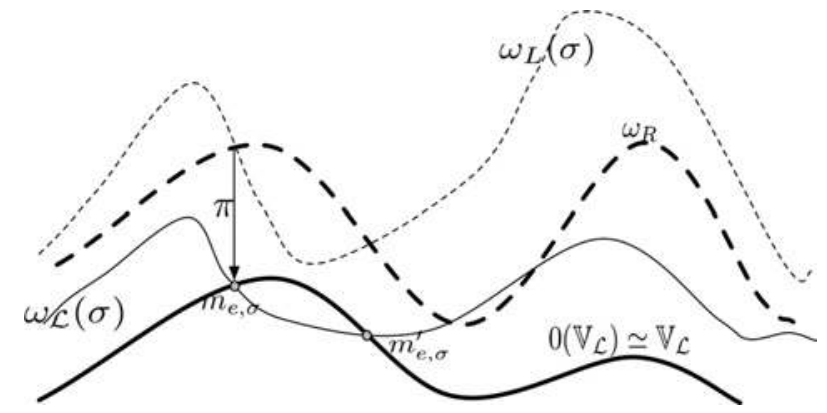

F I G U R E 9 Equivalence between $\omega_{\mathcal{L}}(\sigma) T \mathbb{V}_{\mathcal{L}}$ and $\omega_{L}(\sigma) T \omega_{R}$

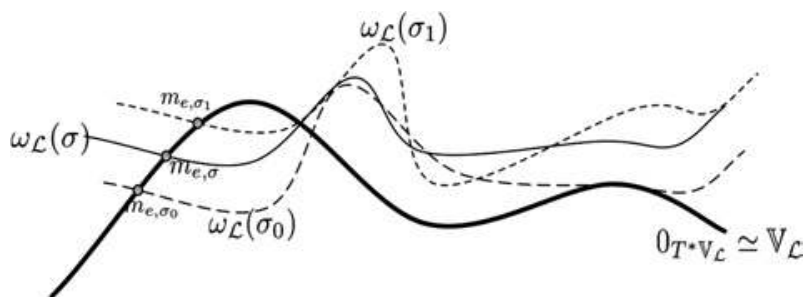

F I G U R E 10 Regular Loading Path $\mathcal{L}=\left(\mathbb{V}_{\mathcal{L}}, \omega_{\mathcal{L}}\right)$

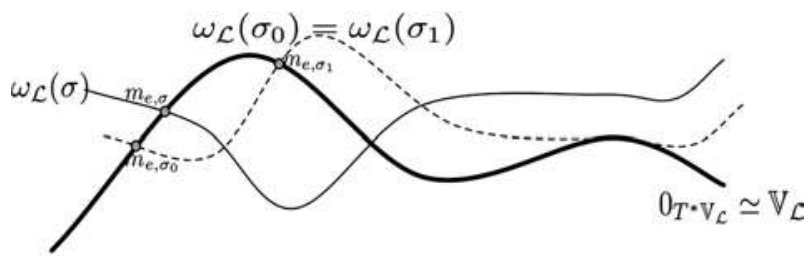

F I G U R E 11 Regular Loading Path with $\omega_{\mathcal{L}}\left(\sigma_{0}\right)=\omega_{\mathcal{L}}\left(\sigma_{1}\right)$

Once this choice is made, the loading path is a map $\sigma \in\left[\sigma_{0}, \sigma_{1}\right] \mapsto \omega_{L}(\sigma) \in \mathfrak{X}^{*}\left(\mathbb{V}_{\mathcal{L}}\right)=\Gamma\left(T^{*} \mathbb{V}_{\mathcal{L}}\right)$ (force part of control). It is then a differentiable one-parameter distribution of sections of $T^{*} \mathbb{V}$. For each step $\sigma$, we also put $\omega_{\mathcal{L}}(\sigma)=\omega_{L}(\sigma)-\omega_{R} \in \mathfrak{X}^{*}\left(\mathbb{V}_{\mathcal{L}}\right)$. Finally, because we investigate only quasi-static evolutions of $\Sigma$ we may always suppose that, at the initial step $\sigma_{0}, \omega_{\mathcal{L}}\left(\sigma_{0}\right)$ transversally intersects the nil section $0_{T^{*} \mathbb{V}_{\mathcal{L}}}$ at a point $m_{e, \sigma_{0}} \in \mathbb{V}_{\mathcal{L}}$ which is the initial equilibrium configuration of the loading path. We then assume that the loading path starts at a stable equilibrium configuration $m_{e, \sigma_{0}}$. This hypothesis will be always implicit.

Definition 10. A loading path $\mathcal{L}$ for the mechanical system $\Sigma$ is

1. a given submanifold $\mathbb{V}_{\mathcal{L}}$ of $\mathbb{M}$

2. a differentiable map $\omega_{L}:\left[\sigma_{0}, \sigma_{1}\right] \subset \mathfrak{X}^{*}\left(\mathbb{V}_{\mathcal{L}}\right)=\Gamma\left(T^{*} \mathbb{V}_{\mathcal{L}}\right)$. We then put $\omega_{\mathcal{L}}=\omega_{L}-\omega_{R}$ namely $\omega_{\mathcal{L}}(\sigma)=\omega_{L}(\sigma)-\omega_{R}$ for all $\sigma \in\left[\sigma_{0}, \sigma_{1}\right]$.

We note $\mathcal{L}=\left(\mathbb{V}_{\mathcal{L}}, \omega_{\mathcal{L}}\right)$ such a loading path.

Definition 11. $\mathcal{L}=\left(\mathbb{V}_{\mathcal{L}}, \omega_{\mathcal{L}}\right)$ is a regular loading path when $\omega_{\mathcal{L}}(\sigma)$ intersects transversally the nil section for all $\sigma \in\left[\sigma_{0}, \sigma_{1}\right]$. The current intersection point is then denoted by $m_{e, \sigma}$.

This condition is equivalent to $\omega_{L}(\sigma)$ intersects transversally $\omega_{R}$ for all $\sigma \in\left[\sigma_{0}, \sigma_{1}\right]$. Figure 9 illustrates these both equivalent points of view. For the rest of the paper, the reasonings and the illustrations are done with $\omega_{\mathcal{L}}$ and the nil section identified with the submanifold itself..

The picture (10) illustrates a regular loading path $\mathcal{L}=\left(\mathbb{V}_{\mathcal{L}}, \omega_{\mathcal{L}}\right)$. From now on, we intentionally omitted the fibers on the pictures.

On the Figure 10, the curve $\sigma \mapsto m_{e, \sigma}$ of equilibrium configurations is obtained for an injective map $\sigma \mapsto \omega_{\mathcal{L}}(\sigma)$. This is not necessary as depicted on Figure 11 where for example $\omega_{\mathcal{L}}\left(\sigma_{0}\right)=\omega_{\mathcal{L}}\left(\sigma_{1}\right)$. 


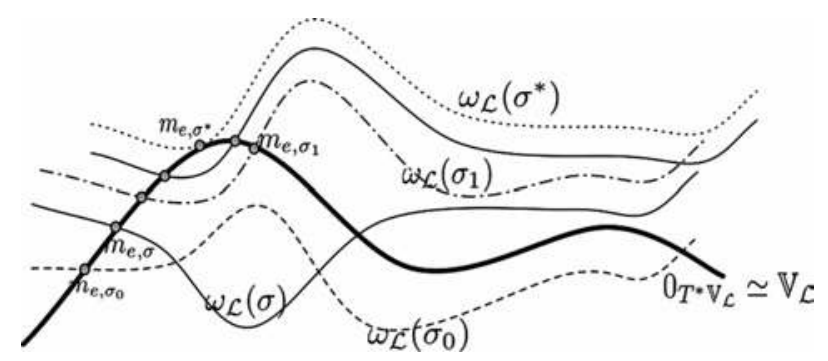

F I G U R E 12 Singular Loading Path $\mathcal{L}$ with the limit loading $\omega_{\mathcal{L}}\left(\sigma^{*}\right)$ and the corresponding instable equilibrium $m_{e, \sigma^{*}}$

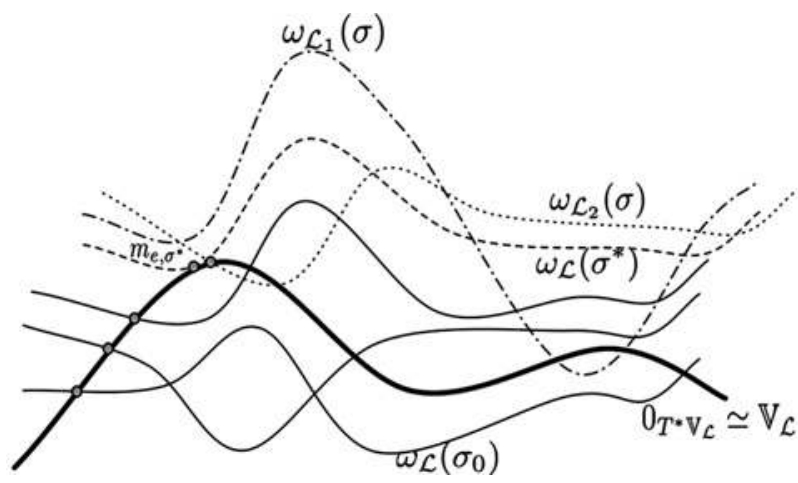

F I G U R E 13 Singular Loading Path with the limit loading $\omega_{\mathcal{L}}\left(\sigma^{*}\right)$ and two corresponding continuations $\omega_{\mathcal{L}_{1}}$ and $\omega_{\mathcal{L}_{2}}$

The transversality condition is preserved by continuous deformations. It means that when $\omega_{\mathcal{L}}(\sigma)$ intersects transversally the nil section for $\sigma \in\left[\sigma_{0}, \sigma_{1}\right]$, then there is an interval $] \sigma-\epsilon, \sigma+\epsilon$ [ such that $\omega_{\mathcal{L}}\left(\sigma^{\prime}\right)$ intersects transversally the nil section for $\left.\sigma^{\prime} \in\right] \sigma-\epsilon, \sigma-\epsilon$ [. In other words, the set of $\sigma$ such that $\omega_{\mathcal{L}}\left(\sigma^{\prime}\right)$ intersects transversally the nil section is an open set $U_{T}$ of $\left[\sigma_{0}, \sigma_{1}\right]$. Because $\omega_{\mathcal{L}}\left(\sigma_{0}\right)$ intersects transversally the nil section at $m_{e, \sigma_{0}}$ we may consider the connexe component of $\sigma_{0}$ in $U_{T}$. It is an interval $\left[\sigma_{0}, \sigma^{*}[\right.$.

Two possibilities may occur $\sigma^{*}=\sigma_{1}$ and then the loading path might be considered as regular. The interesting case is when $\sigma^{*}<\sigma_{1}$ namely when, at the step $\sigma^{*}, \omega_{\mathcal{L}}\left(\sigma^{*}\right) X^{m_{e, \sigma^{*}}} 0_{T^{*} \mathbb{V}_{\mathcal{L}}}$. Sometimes, it could theoretically be possible to continue the loading path $\mathcal{L}$ beyond $\sigma^{*}$ as illustrated on Figure 12. But concretely, the T-instability of $m_{e, \sigma^{*}}$ stops the tracking of the loading path. We then consider such a $\omega_{\mathcal{L}}\left(\sigma^{*}\right)$ as a limit point of the loading path $\mathcal{L}$. Such a loading path is called a singular loading path.

To conclude with the regular/ singular equilibrium configurations, it is worth noting that the 'direction' of the loading path namely the deformation of the load parameters to progress along the 'story' of the loading is locally not significant for the possibility to progress when the transversality condition holds. On the contrary, for a limit loading as $\omega_{\mathcal{L}}\left(\sigma^{*}\right)$ on Figure 13, one can find a loading continuation $\omega_{\mathcal{L}_{1}}$ without any corresponding equilibrium and another loading continuation $\omega_{\mathcal{L}_{2}}$ that allows a quasi static progress. The similarity with Thom's catastrophe theory and the structural stability is obvious. These issues may be viewed as post-critical behavior questions. As already above mentioned, they are not tackled in this paper, especially because dynamic considerations cannot be a priori omitted in such investigations.

\section{7 | KINEMATIC STRUCTURAL STABILITY}

We just above mentioned Thom's catastrophe theory. It is worth noting that transversality is a crucial notion in Thom's works as well as the so-called structural stability. For example, in the sense of Thom's idea, the stability of an equilibrium configuration $m_{e, L}$ for the system $\Sigma$ subjected to the loading $\omega_{L}$ means that there is another close equilibrium when the load system $\omega_{L}$ is slightly changed or perturbed. It is a form of structural stability. Referring now to the loading path concept, it is easy to be convinced that, when the submanifold $\mathbb{V}$ of $\mathbb{M}$ is fixed, the set $\Omega_{\mathbb{V}, \text { reg }}=\left\{\mathcal{L} \mid \mathbb{V}_{\mathcal{L}}=\mathbb{V}\right.$ and $\mathcal{L}$ is regular $\}$ is an open set of $\Gamma\left(T^{*} \mathbb{V}\right)$ for a suitable topology. It is another form of structural stability.

Now, in this section, another type of perturbation is considered. According to the definition of a loading path $\mathcal{L}$, another way to perturb the system is to change the kinematic constraints that means here to change the submanifold $\mathbb{V}_{\mathcal{L}}$ defining the loading path. By changing, we mean adding new kinematic constraints or equivalently choosing a submanifold of $\mathbb{V}_{\mathcal{L}}$. As mentioned in the introduction, such a problem has been already dealt with in the last years but in the linear "punctual" framework (see [16] 
for an overview). Figure 7 perfectly shows what means "punctual": the curve $\gamma: \sigma \mapsto m_{e, \sigma}=\pi\left(\omega_{\mathcal{L}}(\sigma)\right)$ is reduced to a unique point $m_{e} \in \mathbb{V}$. It is the reason why the investigations about KISS could then be led with tools coming only from linear algebra. Here, the general framework is addressed. We now translate the KISS issue into the vector bundle and loading path languages.

Let now $\mathcal{L}=\left(\mathbb{V}_{\mathcal{L}}, \omega_{\mathcal{L}}\right)$ be a fixed regular loading path and $\mathbb{W}$ be an embedded submanifold of $\mathbb{V}_{\mathcal{L}}$ and thus, of $\mathbb{M}$. Does $\mathcal{L}=\left(\mathbb{V}_{\mathcal{L}}, \omega_{\mathcal{L}}\right)$ canonically induce a regular loading path $\mathcal{L}_{\mathbb{W}}=\left(\mathbb{W}, \omega_{\mathcal{L}_{\mathbb{W}}}\right)$ on $\mathbb{W}$ ?

To answer this question, we first must specify what means that $\mathcal{L}$ induces $\mathcal{L}_{\mathbb{W}}$. The obvious first condition in order to correctly define such an induction of $\mathcal{L}_{\mathbb{W}}$ by $\mathcal{L}$ is that the (image of the) curve $\gamma: \sigma \mapsto m_{e, \sigma}=\pi\left(\omega_{\mathcal{L}}(\sigma)\right)$ lies on $\mathbb{W}$. If not, there is no chance to can compare the (T-)stability of equilibrium configurations subjected to kinematic constraints. It is the source of several misunderstandings like those regarding Tarnai's papers where critical loadings for different equilibriums are compared (see [23] for example). From now on, we suppose that this condition always holds in our reasonings.

We have first to define $\omega_{\mathcal{L}_{\mathbb{W}}} \cdot \omega_{\mathcal{L}_{\mathbb{W}}}$ is the "restriction" of $\omega_{\mathcal{L}}$ to $\mathbb{W}$ in the following sense: if $\sigma$ is a step of the loading path and $w \in \mathbb{W}$, then $\omega_{\mathcal{L}_{\mathbb{W}}}(\sigma)(w)(X)=\omega_{\mathcal{L}}(\sigma)(w)(X) \forall X \in T_{w} \mathbb{W} \subset T_{w} \mathbb{V}_{\mathcal{L}}$. In mathematical terms, $\omega_{\mathcal{L}_{\mathbb{W}}}$ is the pull-back $i_{\mathbb{W}}^{*}\left(\omega_{\mathcal{L}}\right)$ of the section $\omega_{\mathcal{L}}$ by the embedding $i_{\mathbb{W}}: \mathbb{W} \rightarrow \mathbb{V}_{\mathcal{L}}$. It follows that $i_{\mathbb{W}}^{*}\left(\omega_{\mathcal{L}}\right)=\omega_{\mathcal{L}_{\mathbb{W}}}$ is a section of the pull-back vector bundle $i_{\mathbb{W}}^{*}\left(T^{*} \mathbb{V}_{\mathcal{L}}\right)=T^{*} \mathbb{W}$ of $T^{*} \mathbb{V}_{\mathcal{L}}$ by the embedding $i_{\mathbb{W}}: \mathbb{W} \rightarrow \mathbb{V}_{\mathcal{L}}$.

Definition 12. The corresponding loading path $\mathcal{L}_{\mathbb{W}}$ is then called a subloading path of $\mathcal{L}_{\mathbb{V}}=\mathcal{L}$.

We then deduce that, for any $\sigma \in\left[\sigma_{0}, \sigma_{1}\right]$, the tangent stiffness $(0,2)$-tensor $d \omega_{\mathcal{L}_{\mathbb{W}}}(\sigma)^{v e r}\left(m_{e, \sigma}\right)$ is the restriction of $d \omega_{\mathcal{L}}(\sigma)^{v e r}\left(m_{e, \sigma}\right)$ to $T_{m_{e, \sigma}} \mathbb{W}$. This object corresponds to the so-called compression of linear operators which has been introduced in previous papers to investigate the linear "punctual" case (see [13] for example).

Theorem 4. Let $\mathcal{L}$ be a regular loading path and $m_{e, \sigma}=\pi\left(\omega_{\mathcal{L}}(\sigma)\right) \in \mathbb{V}_{\mathcal{L}}$. If $d \omega_{\mathcal{L}}(\sigma)^{\text {ver }, s}\left(m_{e, \sigma}\right)$ is a degenerated $(0,2)$ symmetric tensor then there is a submanifold $\mathbb{W} \ni m_{e, \sigma}$ of $\mathbb{V}_{\mathcal{L}}$ such that $\mathcal{L}_{\mathbb{W}}=\left(\mathbb{W}, \omega_{\mathcal{L}_{\mathbb{W}}}\right)$ is singular at $\sigma$.

Proof. Let $m_{e, \sigma}=\pi\left(\omega_{\mathcal{L}}(\sigma)\right) \in \mathbb{V}_{\mathcal{L}}$ be a (regular) point of $\mathcal{L}$ such that $d \omega_{\mathcal{L}}(\sigma)^{\text {ver,s }}\left(m_{e, \sigma}\right)$ is a degenerated symmetric $(0,2)$-tensor. Let $X \neq 0 \in T_{m_{e, \sigma}} \mathbb{V}_{\mathcal{L}}$ be such that

$$
d \omega_{\mathcal{L}}(\sigma)^{\text {ver,s}}\left(m_{e, \sigma}\right)(X, X)=0
$$

Let then $\mathbb{W}$ be any $\left(\operatorname{dim} \mathbb{V}_{\mathcal{L}}-1\right)$ dimensional submanifold of $\mathbb{V}_{\mathcal{L}}$ whose tangent space at $m_{e, \sigma}$ is $\operatorname{Ker} d \omega_{\mathcal{L}}(\sigma)^{\operatorname{ver}}\left(m_{e, \sigma}\right)(X)$. Thus

$$
d \omega_{\mathcal{L}}(\sigma)^{v e r}\left(m_{e, \sigma}\right)(X)(X)=d \omega_{\mathcal{L}}(\sigma)^{v e r}\left(m_{e, \sigma}\right)(X, X)=d \omega_{\mathcal{L}}(\sigma)^{v e r, s}\left(m_{e, \sigma}\right)(X, X)=0
$$

which means that $X \in T_{m_{e, \sigma}} \mathbb{W}$.

Then $d \omega_{\mathcal{L}_{\mathbb{W}}}(\sigma)^{\operatorname{ver}}\left(m_{e, \sigma}\right)$ is not a bijective linear map from $T_{m_{e, \sigma}} \mathbb{W}$ onto $T_{m_{e, \sigma}}^{*} \mathbb{W}$ because by construction, $d \omega_{\mathcal{L}_{\mathrm{W}}}(\sigma)^{\operatorname{ver}}\left(m_{e, \sigma}\right)(X)=0$ whereas $X \neq 0$.

The reciprocal statement also holds:

Theorem 5. Let $\mathcal{L}=\left(\mathbb{V}_{\mathcal{L}}, \omega_{\mathcal{L}}\right)$ be a regular loading path such that $d \omega_{\mathcal{L}}(\sigma)^{\text {ver,s }}\left(m_{e, \sigma}\right)$ is a positive non degenerated $(0,2)$ symmetric tensor for any $\sigma$. Then for any submanifold $\mathbb{W}$ of $\mathbb{V}_{\mathcal{L}}, \mathcal{L}_{\mathbb{W}}=\left(\mathbb{W}, \omega_{\mathcal{L}_{\mathbb{W}}}\right)$ is a regular loading path.

To sum up this section, the KISS issue has been extended from the punctual case of a single stable equilibrium position to the general case of regular loading path. The main result making a full bridge between T-stability and Hill-stability has been extended: in order that a regular loading path $\mathcal{L}=\mathcal{L}_{\mathbb{V}}=\left(\mathbb{V}_{\mathcal{L}}, \omega_{\mathcal{L}}\right)$ has only regular subloading paths $\mathcal{L}_{\mathbb{W}}$, it is necessary and sufficient that $d \omega_{\mathcal{L}}(\sigma)^{v e r, s}\left(m_{e, \sigma}\right)$ is a positive non degenerated $(0,2)$ symmetric tensor for any $\sigma$ : the regular (namely T-stable) loading path $\mathcal{L}$ is Hill-stable.

\section{I EXAMPLE}

In this section, the 4 spheres granular system called the diamond pattern illustrates the above general developments. [19] provides an accurate investigation of this granular system with both elastic and elastic-plastic behaviors. To be in accordance with the framework of the above investigations, only the elastic behavior will be investigated. We roughly take the notations of [19] but we adapt them to the present geometrical framework and we follow the same order than for this general framework: kinematics, loading forces, link forces, equilibriums and constitutive laws.

It is worth emphasizing that this example is proposed only for the sake of illustration of the general approach. This example is used because it leads to a "simple" non conservative system with a non symmetric stiffness operator in the framework of 
granular systems which is a well appropriate framework for illustrating the nonconservativeness of the system. Furthermore, it is conceivable that different methods exist to tackle this four grains system. As any discrete model of granular media, there are for example great similarities with other mechanical systems like bars and cables systems used in architecture design especially investigated through the tensegrity approach (see for example [6,7]) or through tools of the mechanism theory and more specifically with the so-called infinitesimal investigations (see for example [2,10]) and the links with possible finite mobility.

In the tensegrity approach, the idea is to build or design a system of bars and cables self balancing by a play between tensions and compressions in the involved elements of the structure. A similarity with the problem of the diamond pattern does certainly exist as for other systems exhibited in granular media. However, a large part of the investigations of granular systems address the contact laws between the elements which may introduce a plastic behavior of the system. Moreover, the investigated systems are not designed by human beings but produced by the nature. We then observe that only of few type of characteristic systems are exhibited by the granular media whereas the rules of the tensegrity approach give rise to a variety of mechanical structures.

Regarding the possible use of mechanism theory, we take the opportunity to provide further details about the deep issue of the links between infinitesimal and finite mobility as discussed by Kuznetzov for example in [10] devoted to the links with statics and especially with the statical indeterminacy. The author underlines the key rule of quadratic and higher terms in kinematic relations for possible (paradoxical) infinitesimal higher order mobility but without highlighting the mechanical meaning of these terms which must be evaluated for each mechanical system. In the same years (in 1987 for his $\mathrm{PhD}$ thesis), the first author of the present paper provided new results regarding these difficulties and paradoxes by use of calculations on Lie groups. The so-called first and higher order mobilities introduced by Kuznetsov had then been directly and explicitly connected to appropriate combinations of Lie brackets of vectors of each Lie algebra attached to the links of the mechanisms. Following these investigations, a full classification of singularities of mechanisms has been proposed in a founding paper in 1998 [9] which extended the solution of the problem of the infinitesimal motions to singular situations by use of analytic geometry and the computation of the tangent cone at a singularity. It is interesting to note that the transversality concept is also the crux concept to tackle the singular mechanisms even tough it it then used in another complete different way than in the present paper.

To conclude about these developments, the mechanism theory is especially interesting for singularity analysis. In the present paper, the kinematics is taken into account only as a given regular differentiable manifold $\mathbb{V}$ without singularity. The kinematic constraints which have to be first investigated in order to derivate charts of $\mathbb{V}$ are then supposed - at least locally- regular and we directly start with such charts of $\mathbb{V}$ like the charts $(\ell, \alpha)$ or $\left(L_{1}, L_{2}\right)$ in the next paragraph. An accurate analysis of the derivation of such charts is out of the scope of the paper and, on the contrary, we focus on general constitutive laws or loading that may lead to non symmetric tangent stiffness operators.

\section{1 | Kinematics}

To focus on the involved geometrical features, some modifications are done with respect to the developments of [19]. The diamond pattern is a 4 spheres granular system. To describe it, we have mainly to describe firstly its kinematics and secondly its behavior namely the constitutive behavior of the contact between two grains. The system is supposed to be in the plane $\left(O, x_{1}, x_{2}\right) .4$ free spheres in the plane mean a 12 degrees of freedom system. The contact model suppose that there are no proper rotation of the spheres which leads to 8 degrees of freedom. Now the symmetry of the loading system means that the center of inertia of the whole system is fixed $(\equiv O)$ so that the degree of freedom drops to 6 . The symmetry of the loading system also implies that the system of the centers (of inertia) of the spheres always have a diamond shape. Finally, the diamond pattern is a 2 dof system and the configuration space $\mathbb{M}$ is a two dimension manifold. This discussion shows that the initial manifold $\mathbb{M}$ before choosing more particular loading paths is itself a submanifold of a larger manifold. Because no rotation of the spheres is possible, each sphere $\left(S_{i}, i=1,2,3,4\right)$ may be viewed as a point $G_{i}, i=1,2,3,4$ (see Figure 14) from a kinematic point of view. Two natural charts $\left(L_{1}, L_{2}\right)=\phi_{1}(m)$ and $(d, \alpha)=\phi_{2}(m)$ are possible to describe the configuration $m \in \mathbb{M}$. Indeed, the positions of each $G_{i}, i=1,2,3,4$ is then known. These charts read $\overrightarrow{G_{1} G_{3}}=L_{1} \overrightarrow{x_{1}}, \overrightarrow{G_{2} G_{4}}=L_{2} \overrightarrow{x_{2}}$ and $\overrightarrow{G_{1} G_{2}}=\ell \sin \alpha \overrightarrow{x_{1}}-\ell \cos \alpha \overrightarrow{x_{2}}$ in appropriate open sets of $\mathbb{R}^{2} . \ell$ is the distance between two adjoining spheres which does not depend on the spheres by means of symmetry. In order not to be confused by the loss of rotations of spheres, only the centers of the spheres are drawn and the lines between the center of two adjoining spheres are representations of the links between these corresponding spheres that are supposed perfectly rigid but overlap at the same time. Beyond an attempt to provide a full physical meaning of the mechanical model that is always limited and questionable, the model may be justified by its fruitfulness and the consistency of its results.

$$
\left\{\begin{array}{l}
L_{1}=2 \ell \sin \alpha \\
L_{2}=2 \ell \cos \alpha
\end{array}\right.
$$




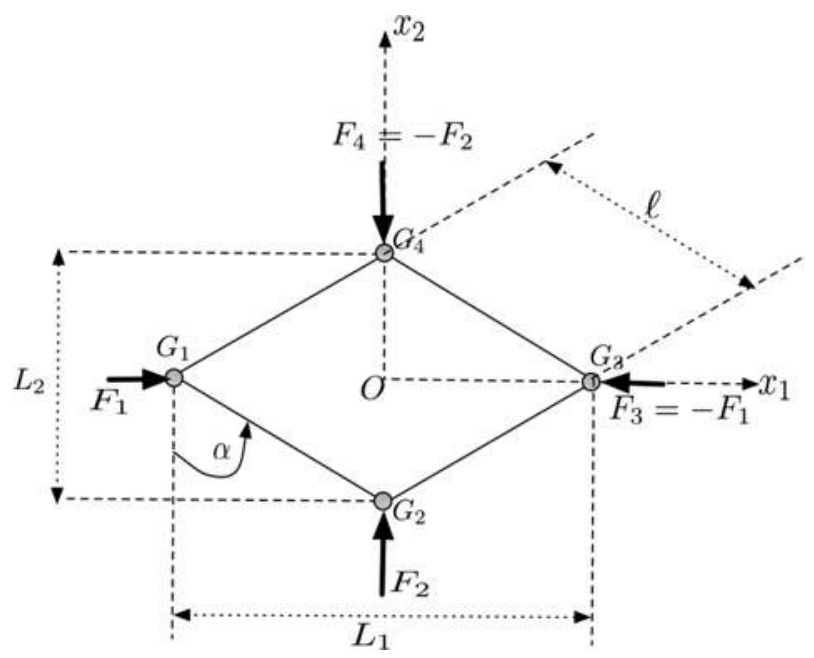

F I G U R E 14 Diamond pattern

describes the change of charts $\phi_{21}=\phi_{1} \circ \phi_{2}^{-1}$ whereas

$$
\left\{\begin{array}{l}
\ell=\frac{1}{2} \sqrt{L_{1}^{2}+L_{2}^{2}} \\
\alpha=\arctan \frac{L_{1}}{L_{2}}
\end{array}\right.
$$

describes the change of charts $\phi_{12}=\phi_{2} \circ \phi_{1}^{-1}=\phi_{21}^{-1}$. (14) is equivalent to

$$
\left\{\begin{aligned}
\ell & =\frac{1}{2} \sqrt{L_{1}^{2}+L_{2}^{2}} \\
\cos \alpha & =\frac{L_{2}}{\sqrt{L_{1}^{2}+L_{2}^{2}}} \\
\sin \alpha & =\frac{L_{1}}{\sqrt{L_{1}^{2}+L_{2}^{2}}}
\end{aligned}\right.
$$

\section{2 | Loading forces}

The load $F=\left\{F_{1}, F_{2}\right\}$ as depicted on Figure 14 leads to the following section $\omega_{F}$ of $T^{*} \mathbb{M}$ with the local coordinates $\left(L_{1}, L_{2}\right)$ :

$$
\omega_{F}\left(L_{1}, L_{2}\right)=-F_{1} d L_{1}-F_{2} d L_{2}
$$

whereas in the second local coordinates $(\ell, \alpha)$, it reads:

$$
\omega_{F}(\ell, \alpha)=-2\left(F_{1} \sin \alpha+F_{2} \cos \alpha\right) d \ell-2 \ell\left(F_{1} \cos \alpha-F_{2} \sin \alpha\right) d \alpha
$$

If $F_{1}$ and $F_{2}$ are constant, then $\omega_{F}$ has a potential $U_{F}(m)=U_{F}\left(L_{1}, L_{2}\right)=F_{1} L_{1}+F_{2} L_{2}$ in the local coordinate system $\left(L_{1}, L_{2}\right)$ and $U_{F}(m)=U_{F}(\ell, \alpha)=2 \ell\left(F_{1} \sin \alpha+F_{2} \cos \alpha\right)$ in the local coordinate system $(\ell, \alpha)$.

\section{3 | Link forces}

Let $m$ be a current configuration of the system described by the coordinate system $\left(L_{1}, L_{2}\right)$ or/and $(\ell, \alpha)$. Before expressing the global constitutive law of the system, we first express the value of the above $\omega_{\ell}(m)$. 
We suppose that the loop of the link graph is oriented as depicted on 14. Let $\left\{\ell_{12}, \ell_{23}, \ell_{34}, \ell_{41}\right\}$ be the set of all oriented links of the system. The contact law of each link $\ell_{i j}$ is described by a couple $\left(N_{i j}, T_{i j}\right)$ of forces so defined. $\left(N_{i j}, T_{i j}\right)$ are the normal and tangential forces of the action of $S_{j}$ on $S_{i}$. Thus, the action of $S_{j}$ on $S_{i}$ reads $\vec{F}_{i j}=-N_{i j} \vec{u}_{i j}+T_{i j} \vec{v}_{i j}$ where $\vec{u}_{i j}$ is the unit vector along $\overrightarrow{G_{i} G_{j}}$ and $\vec{v}_{i j}$ oriented unit orthogonal to $\vec{u}_{i j}$. By convention, the normal action is positive in compression.Thus, by means of action reaction law, this only involves 8 forces $\left\{N_{12}, T_{12}, N_{23}, T_{23}, N_{34}, T_{34}, N_{41}, T_{41}\right\}$. Furthermore, by means of symmetry of the diamond pattern and of the lack of global translation and rotation of the system, this set of unknowns is reduced to only two unknowns $(N, T)$ with $\left(N=N_{12}, T=T_{12}\right)$. The four link forces $\vec{F}_{\ell, i}$ acting on $G_{i}$ for $i=1, \ldots, 4$ read

$$
\begin{aligned}
\vec{F}_{\ell, 1}= & \vec{F}_{12}+\vec{F}_{14} \\
= & \left(\left(-N_{12}-N_{41}\right) \sin \alpha+\left(T_{12}-T_{41}\right) \cos \alpha\right) \vec{x}_{1} \\
& +\left(\left(N_{12}-N_{41}\right) \cos \alpha+\left(T_{12}+T_{41}\right) \sin \alpha\right) \vec{x}_{2} \\
\vec{F}_{\ell, 2}= & \vec{F}_{21}+\vec{F}_{23} \\
= & \left(\left(N_{12}-N_{23}\right) \sin \alpha+\left(-T_{12}-T_{23}\right) \cos \alpha\right) \vec{x}_{1} \\
& +\left(\left(-N_{12}-N_{23}\right) \cos \alpha+\left(-T_{12}+T_{23}\right) \sin \alpha\right) \vec{x}_{2} \\
\vec{F}_{\ell, 3}= & \vec{F}_{32}+\vec{F}_{34} \\
= & \left(\left(N_{23}+N_{34}\right) \sin \alpha+\left(T_{23}-T_{34}\right) \cos \alpha\right) \vec{x}_{1} \\
& +\left(\left(N_{23}-N_{34}\right) \cos \alpha+\left(-T_{23}-T_{34}\right) \sin \alpha\right) \vec{x}_{2} \\
\vec{F}_{\ell, 4}= & \vec{F}_{43}+\vec{F}_{41} \\
= & \left(\left(-N_{34}+N_{41}\right) \sin \alpha+\left(T_{34}+T_{41}\right) \cos \alpha\right) \vec{x}_{1} \\
& +\left(\left(N_{34}+N_{41}\right) \cos \alpha+\left(T_{34}-T_{41}\right) \sin \alpha\right) \vec{x}_{2}
\end{aligned}
$$

The kinematics implies that $\vec{F}_{\ell, 1}+\vec{F}_{\ell, 3}=\overrightarrow{0}=\vec{F}_{\ell, 2}+\vec{F}_{\ell, 4},\left(\vec{F}_{\ell, 1} \mid \vec{x}_{2}\right)=\left(\vec{F}_{\ell, 3} \mid \vec{x}_{2}\right)=0$ and $\left(\vec{F}_{\ell, 2} \mid \vec{x}_{1}\right)=\left(\vec{F}_{\ell, 4} \mid \vec{x}_{1}\right)=0$. Finally, in the second coordinate system $(\alpha, \ell)$, this reads:

$$
\begin{aligned}
\vec{F}_{\ell, 1} & =(-2 N \sin \alpha+2 T \cos \alpha) \vec{x}_{1} \\
\vec{F}_{\ell, 2} & =(-2 N \cos \alpha-2 T \sin \alpha) \vec{x}_{2} \\
\vec{F}_{\ell, 3} & =(2 N \sin \alpha-2 T \cos \alpha) \vec{x}_{1} \\
\vec{F}_{\ell, 4} & =(2 N \cos \alpha+2 T \sin \alpha) \vec{x}_{2}
\end{aligned}
$$

whereas in the first coordinate system $\left(L_{1}, L_{2}\right)$, this reads

$$
\begin{gathered}
\vec{F}_{\ell, 1}=\frac{1}{\sqrt{L_{1}^{2}+L_{2}^{2}}}\left(-L_{1} N+L_{2} T\right) \vec{x}_{1} \\
\vec{F}_{\ell, 2}=\frac{1}{\sqrt{L_{1}^{2}+L_{2}^{2}}}\left(-L_{2} N-L_{1} T\right) \vec{x}_{2} \\
\vec{F}_{\ell, 3}=\frac{1}{\sqrt{L_{1}^{2}+L_{2}^{2}}}\left(L_{1} N-L_{2} T\right) \vec{x}_{1} \\
\vec{F}_{\ell, 4}=\frac{1}{\sqrt{L_{1}^{2}+L_{2}^{2}}}\left(L_{2} N+L_{1} T\right) \vec{x}_{2}
\end{gathered}
$$


Finally, the expression of $\omega_{\ell}(m) \in T^{*} \mathbb{M}$ of all the link forces in the configuration $m$ then reads in the first coordinate system:

$$
\omega_{\ell}(m)=\omega_{\ell}\left(L_{1}, L_{2}\right)=\frac{1}{\sqrt{L_{1}^{2}+L_{2}^{2}}}\left(\left(L_{1} N-L_{2} T\right) d L_{1}+\left(L_{2} N+L_{1} T\right) d L_{2}\right)
$$

whereas in the second local coordinates $(\ell, \alpha)$, it reads:

$$
\omega_{\ell}(m)=\omega_{\ell}(\ell, \alpha)=4 N d \ell-4 T \ell d \alpha
$$

Calculations give $\mathbf{d} \omega_{\ell}(\ell, \alpha)=\left(-4 \frac{\partial N}{\partial \alpha}-4 T-4 \ell \frac{\partial T}{\partial \ell}\right) d \ell \wedge d \alpha$. This shows that, if

$$
\frac{\partial N}{\partial \alpha}+T+\ell \frac{\partial T}{\partial \ell}=0
$$

then $\mathbf{d} \omega_{\ell}=0$ and $\omega_{\ell}$ is closed. Often, $N=N(\ell)$ (and $T=T(\ell, \alpha)$ is any). Thus, in this case, $\omega_{\ell}$ is closed if and only if $T$ is solution of

$$
T+\ell \frac{\partial T}{\partial \ell}=0
$$

whose solution is $T(\ell, \alpha)=\frac{S(\alpha)}{\ell}$ with $S: \alpha \mapsto S(\alpha)$ any function. If not, the system is hypoelastic. We will come back to this issue is the section devoted to the constitutive law (see paragraph 8.5 ).

\section{4 $\mid$ Equilibriums}

Let $F=\left\{F_{1}, F_{2}\right\}$ be a loading system. For this load, equilibrium equations read

$$
\omega_{F}(m)+\omega_{\ell}(m)=0
$$

In the first coordinate system, an equilibrium configuration $m_{e}=\left(L_{e, 1}, L_{e, 2}\right)$ is then such that:

$$
\omega_{F}\left(L_{e, 1}, L_{e, 2}\right)+\omega_{\ell}\left(L_{e, 1}, L_{e, 2}\right)=0
$$

which also reads:

$$
\left(-F_{1}+\frac{1}{\sqrt{L_{e, 1}^{2}+L_{e, 2}^{2}}}\left(L_{e, 1} N-L_{e, 2} T\right)\right) d L_{1}+\left(-F_{2}+\frac{1}{\sqrt{L_{e, 1}^{2}+L_{e, 2}^{2}}}\left(L_{e, 2} N+L_{e, 1} T\right)\right) d L_{2}=0
$$

This is equivalent to the system

$$
\left\{\begin{array}{l}
F_{1}=\frac{1}{\sqrt{L_{e, 1}^{2}+L_{e, 2}^{2}}}\left(L_{e, 1} N-L_{e, 2} T\right) \\
F_{2}=\frac{1}{\sqrt{L_{e, 1}^{2}+L_{e, 2}^{2}}}\left(L_{e, 2} N+L_{e, 1} T\right)
\end{array}\right.
$$

In the second coordinate system, an equilibrium configuration $m_{e}=\left(\ell_{e}, \alpha_{e}\right)$ is then such that:

$$
\omega_{F}\left(\ell_{e}, \alpha_{e}\right)+\omega_{\ell}\left(\ell_{e}, \alpha_{e}\right)=0
$$

which also reads:

$$
2\left(-F_{1} \sin \alpha_{e}-F_{2} \cos \alpha_{e}+2 N\right) d \ell+2 \ell_{e}\left(-F_{1} \cos \alpha_{e}+F_{2} \sin \alpha_{e}-2 T\right) d \alpha=0
$$


This is equivalent to the system

$$
\left\{\begin{array}{r}
F_{1} \sin \alpha_{e}+F_{2} \cos \alpha_{e}=2 N \\
-F_{1} \cos \alpha_{e}+F_{2} \sin \alpha_{e}=2 T
\end{array}\right.
$$

which also reads

$$
\left\{\begin{array}{l}
F_{1}=2 N \sin \alpha_{e}-2 T \cos \alpha_{e} \\
F_{2}=2 N \cos \alpha_{e}+2 T \sin \alpha_{e}
\end{array}\right.
$$

\section{5 | Constitutive laws}

The constitutive law of the system is brought back to the one of each link. Elasticity then means that the mechanics of the links is described by differentiable relations $N=N(m), T=T(m)$ or $N=N\left(L_{1}, L_{2}\right), T=T\left(L_{1}, L_{2}\right)$ in the first coordinate system and $N=N(\ell, \alpha), T=T(\ell, \alpha)$ in the second coordinate system. We already discussed in paragraph 8.3 about the closedness of $\omega_{\ell}$. Except when the condition 20 holds, the constitutive law is hypoelastic and leads to a differentiable fixed section $\omega_{\ell} \in \mathfrak{X}^{*}(\mathbb{M})$ which a non closed 1 -form on $\mathbb{M}$.

However, the incremental formulation of the modeling of this constitutive law of the contact between two spheres is in fact the most appropriate. We now deal with.

Let $m \in \mathbb{M}$ be any configuration and let $\{N, T\}$ be a link forces system so that relation (19) holds. In this state described by such $\omega_{\ell}$ with $\pi\left(\omega_{\ell}\right)=m$, let $(\dot{N}, \dot{T}, \dot{\ell}, \dot{\alpha})$ be a local system of coordinates of any element of $T_{\omega_{\ell}} T^{*} \mathbb{M}$ associated with the local coordinates $(\ell, \alpha)$ at $m$. The subspace $\tau_{e}\left(\omega_{\ell}\right)$ (see Section 5) is defined by the two linear equations

$$
\left\{\begin{array}{l}
\dot{N}+k_{n} \dot{\ell}=0 \\
\dot{T}-k_{t} \ell \dot{\alpha}=0
\end{array}\right.
$$

The distribution $\tau_{e}: \omega_{\ell} \mapsto \tau_{e}\left(\omega_{\ell}\right)$ defines the incremental elastic law of the diamond pattern. $k_{n}$ and $k_{t}$ are two given positive scalars. Let us show that this distribution is not integrable. By Frobenius theorem, we have to compute the Lie braket of the two following generators of $\tau_{e}\left(\omega_{\ell}\right)$. In the second coordinate system $(\ell, \alpha)$, they read $X=\frac{\partial}{\partial \ell}-k_{n} \frac{\partial}{\partial N}, Y=\frac{\partial}{\partial \alpha}+k_{t} \ell \frac{\partial}{\partial T}$. Then, $[X, Y]=k_{t} \frac{\partial}{\partial T}$ which does not belong to the module spanned by $X$ and $Y$. Beyond this systematic proof, it may be obviously remarked that the first equation of (29) is integrable by $N=-k_{n} \ell$ whereas the second one cannot be integrated because $\ell d \alpha$ is not closed and then not exact. As mentioned in [19], the purely geometric property that $\ell d \alpha$ is a non closed form is the source of the nonconservativeness of the diamond system. It is directly reflected here in the non integrability of the chosen constitutive laws because of the linearity of these constitutive laws. The chosen constitutive laws are then non integrable hypoelastic.

\section{6 | Stiffness tensor}

We use again the second coordinate system. Let $m_{e}=\left(\ell_{e}, \alpha_{e}\right) \in \mathbb{M}$ be an equilibrium configuration of the system that undergoes the loading system $F=\left\{F_{1}, F_{2}\right\}$ and the link forces system $\{N, T\}$ so that Equation 28 holds. According to Equation 26,

$$
\omega\left(m_{e}\right)=2\left(-F_{1} \sin \alpha_{e}-F_{2} \cos \alpha_{e}+2 N\right) d \ell+2 \ell_{e}\left(-F_{1} \cos \alpha_{e}+F_{2} \sin \alpha_{e}-2 T\right) d \alpha
$$

Subjected to an incremental perturbation $(\dot{\ell}, \dot{\alpha})$ leads to a response $(\dot{N}, \dot{T})$ of the system so that $(\dot{N}, \dot{T}, \dot{\ell}, \dot{\alpha}) \in F\left(m_{e}\right)$. Thus, $\dot{N}=-k_{n} \dot{\ell}, \dot{T}=k_{t} \ell \dot{\alpha}$. In this local coordinate system, $d \omega^{v e r}\left(m_{e}\right) \in \mathcal{L}\left(T_{m_{e}} \mathbb{M}, T_{m_{e}}^{*} \mathbb{M}\right)$ then reads:

$$
\begin{aligned}
d \omega^{v e r}\left(m_{e}\right)\left(\left.\dot{\ell} \frac{\partial}{\partial \ell}\right|_{m_{e}}+\left.\dot{\alpha} \frac{\partial}{\partial \alpha}\right|_{m_{e}}\right)= & -2\left(-F_{1} \cos \alpha_{e} \dot{\alpha}+F_{2} \sin \alpha_{e} \dot{\alpha}-2 k_{n} \dot{\ell}\right) d \ell \\
& +2 \ell_{e}\left(F_{1} \sin \alpha_{e} \dot{\alpha}+F_{2} \cos \alpha_{e} \dot{\alpha}-2 k_{t} \dot{\alpha} \ell_{e}\right) d \alpha
\end{aligned}
$$

or as a $(0,2)$ tensor :

$$
\begin{aligned}
d \omega^{v e r}\left(m_{e}\right)= & 4 k_{n} d \ell \otimes d \ell+2\left(F_{1} \cos \alpha_{e}+F_{2} \sin \alpha_{e}\right) d \ell \otimes d \alpha \\
& +2 \ell_{e}\left(F_{1} \sin \alpha_{e}+F_{2} \cos \alpha_{e}-2 k_{t} \ell_{e}\right) d \alpha \otimes d \alpha
\end{aligned}
$$


so that the loading $F=\left\{F_{1}, F_{2}\right\}$ leading to divergence instability is such that

$$
F_{1} \sin \alpha_{e}+F_{2} \cos \alpha_{e}-2 k_{t} \ell_{e}=0
$$

The corresponding stiffness matrix (in the local coordinate system $(\ell, \alpha)$ ) reads

$$
K\left(\ell_{e}, \alpha_{e}\right)=\left(\begin{array}{cc}
4 k_{n} & 2\left(F_{1} \cos \alpha_{e}+F_{2} \sin \alpha_{e}\right) \\
0 & 2 \ell_{e}\left(F_{1} \sin \alpha_{e}+F_{2} \cos \alpha_{e}-2 k_{t} \ell_{e}\right)
\end{array}\right)
$$

Finally, as mentioned and deeply investigated in [19], this stiffness tensor is not symmetric which signs the nonconservativeness of the system. This then makes sense to use the Hill criterion as in [19].

\section{CONCLUSION}

In this paper, we follow our goal to expand to the non linear framework and by a geometric and intrinsic way the set of concepts and results of these six last past years. The two main new concepts introduced to investigate nonconservative systems have been the KISS and the GDNC. The extension of GDNC has already been achieved in one of latest publications thanks to the exterior calculus on manifolds and some of the tools of symplectic geometry (see [15]). Here, the KISS problem as well as a great part of associated results are extended from the linear "punctual" framework to any non linear discrete framework. One of the major obstacle to directly perform this extension has been to capture the intrinsic picture of the so-called tangent stiffness matrix from which the stability analysis of the evolution is done. The usual splitting of the stiffness matrix $K$ into its symmetric $K_{s}$ and skewsymmetric parts $K_{a}$ had been the key tools to tackle respectively the KISS and GDNC issues. Whereas the exterior derivative $\mathbf{d} \omega$ was the natural extension of $K_{a}$ to the non punctual and non linear framework, no natural tool seemed emerge for extending $K_{s}$. In fact and surprisingly, the skew symmetric part emerged more naturally than the whole tensor field corresponding to $K$. The deep reason was that the whole tensor field should correspond to a derivative of a section of a vector bundle (here $\omega$ ) that can been performed only through a connection on the involved vector bundle. But there is no natural connection associated to our problem. Indeed, for a mechanical problem, the usual connection is the one of the Riemanniann structure associated to the kinetic energy. Here, the goal to investigate quasi-static or incremental evolution of the mechanical system removes any resort to kinetic energy. By using the fact that the evolution lies on the nil section, we bypass the lack of connection on the vector bundle and we perform the claimed goal: the stability is pulled back to the transversality to a manifold, the intrinsic tangent stiffness tensor is the vertical component $d \omega^{v e r}$ of the differential $d \omega$ which is meaningful only on the nil section because of the lack of connection. In a second time, the usual characterization of the stability by the invertibility of $d \omega^{\text {ver }}$ becomes a theorem. Hill's second order work criterion is translated in this language and its first link with stability of equilibrium is carried out. General non linear loading paths $\mathcal{L}$ are then defined as couples $\mathcal{L}=\left(\mathbb{V}_{\mathcal{L}}, \omega_{\mathcal{L}}\right)$ with $\mathbb{V}_{\mathcal{L}}$ embedded submanifold of $\mathbb{M}$ and $\omega_{\mathcal{L}} \in \mathfrak{X}^{*}\left(\mathbb{V}_{\mathcal{L}}\right)=\Gamma\left(T^{*} \mathbb{V}_{\mathcal{L}}\right)$. Such a loading path $\mathcal{L}$ is then the extension of "punctual" equilibrium positions as those investigated during the past years regarding the KISS issue. Regular and singular loading paths have been first studied before dealing with the non linear KISS issue. KISS of regular paths is formulated in the vector bundle language and the equivalence between Hill-stability and T-stability for KISS issue is also extended for any regular loading path. An intrinsic geometric description of integrable and not integrable hypoelasticity is also formulated. A detailed investigation of the granular diamond pattern illustrates these general developments. Plasticity will be the natural extension of the present work and will be tackled with the same spirit in forthcoming papers.

\section{ACKNOWLEDGMENTS}

We would take the opportunity of this work to pay warm tribute to Professor Alain Rigolot who left us last February. Alain Rigolot, with a great generosity and a real intuition, suggested fifteen years ago to the first author to investigate non conservative elastic systems and especially their stability. They published together only one paper (in 2009) on this topic but, during the last ten years, he accurately followed the numerous developments and results on this subject. The present paper is especially dedicated to him.

\section{REFERENCES}

[1] B. Bernstein, Hypo-Elasticity and Elasticity, J. Ration. Mech. Anal. 1960, 6, 89.

[2] D. Chevallier, J. Lerbet, Rigid-body Kinematics and Dynamics with Lie groups, Iste-Wiley, UK 2018.

[3] M. Crampin, F. A. E. Pirani, Applicable Differential Geometry, London Mathematical Society Lecture Note Series. 59, Cambridge U.P. 1994. 
[4] C. Godbillon, Géométrie différentielle et Mécanique Analytique, Col. Méthodes, Hermann, Paris 1969.

[5] N. Challamel, F. Nicot, J. Lerbet, F. Darve, Stability of non-conservative elastic structures under additional kinematics constraints, Engineering Structures 2010, 32, 3086.

[6] M. Hidenori, Static and dynamic analyses of tensegrity structures. Part II. Quasistatic analysis, Int. J. Solids Struct. $2001,38,3615$.

[7] K. Kebiche, M. N. Kazi-Aoual, R. Motro, Geometrical non-linear analysis of tensegrity systems, Engineering Structures 1999, $21,864$.

[8] B. Kolev, Eléments de géométrie différentielle à l'usage des mécaniciens, 5i Ecole d'Eté de Mécanique Théorique de Quiberon, 2016, www.mgm.ida.upmc.fr.

[9] J. Lerbet, Analytic Geometry and Singularities of Mechanisms, Z. Angew. Math. Mech. 1998, 78, 687.

[10] E. N. Kuznetsov, Underconstrained Structural Systems, Int. J. Solids Struct. 1988, 24, 153.

[11] J. Lerbet, M. Aldowaji, N. Challamel, F. Nicot, F. Prunier, F. Darve, P-positive definite matrices and stability of nonconservative systems, Z. Angew. Math. Mech. 2012, 92, 409.

[12] J. Lerbet, N. Challamel, O. Kirillov, F. Nicot, F. Darve, Geometric degree of Non-conservativity, Math. and Mech. of Complex Systems $2014,2$.

[13] J. Lerbet, N. Challamel, F. Nicot, F. Darve, Variational Formulation of Divergence Stability for constrained systems, Appl. Math. Model. 39, 7469.

[14] J. Lerbet, G. Hello, N. Challamel, F. Nicot, F. Darve, 3-Dimensional Flutter Kinematic Structural Stability, Nonlinear Anal., Real World Appl. 2016, 29, 19.

[15] J. Lerbet, N. Challamel, F. Nicot, F. Darve, Geometric Degree of Nonconservativity: set of solutions for the linear case and extension to the differentiable non linear case, Appl. Math. Model. 2016, 40, 5930.

[16] J. Lerbet, N. Challamel, F. Nicot, F. Darve, Kinematical Structural Stability, Discrete and Continuous Dynamical Systems - Series S (DCDS-S) of American Institute of Mathematical Sciences (AIMS), DCDS-S 9-2 June 2016 special issue.

[17] J. Lerbet, N. Challamel, F. Nicot, F. Darve, On the stability of nonconservative continuous systems under kinematic constraints, Z. Angew. Math. Mech. 1-20 (2017) / https://doi.org/10.1002/zamm.201600203.

[18] A. A. Movchan, The direct method of Lyapunov in stability problems of elastic systems, PMM, J. Appl. Math. Mech. 1959, $23,686$.

[19] F. Nicot, G. Veylon, H. Zhu, J. Lerbet, F. Darve, Mesoscopic scale instability in particulate materials, J. Eng. Mechanics 2016, 142, 1, https://doi.org/10.1061/(ASCE)EM.1943-7889.0001100.

[20] F. Nicot, J. Lerbet, F. Darve, Second-order work criterion: from material point to boundary value problems, Acta Mech., accepté pour publication, 2017.

[21] G. Sardanashvily, Fibre Bundles, Jet manifolds and Lagrangian Theory. Lectures for Theoricians, arXiv:0908.1886v2 [math-ph]

[22] J.-M. Souriau, Structure of Dynamical Systems, Progress in Mathematics, Birkhäuser 1997.

[23] T. Tarnai, Paradoxical behaviour of vibrating systems challenging Rayleigh's theorem, 21st International Congress of Theoretical and Applied Mechanics, Warsaw 2004.

[24] C. Truesdell, Hypo-elasticity, J. Rational Mech. Anal. 1955, 4, 83.

[25] C. Truesdell, W. Noll, The non-linear field theories of mechanics, Handbuch der Physik III/c. Springer-Verlag 1965. 